\title{
Bitter and sweet taste receptors regulate human upper respiratory innate immunity
}

\author{
Robert J. Lee, ${ }^{1}$ Jennifer M. Kofonow, ${ }^{1}$ Philip L. Rosen, ${ }^{1}$ Adam P. Siebert, ${ }^{1}$ \\ Bei Chen, ${ }^{1}$ Laurel Doghramji, ${ }^{1}$ Guoxiang Xiong, ${ }^{2}$ Nithin D. Adappa, ${ }^{1}$ James N. Palmer, ${ }^{1}$ \\ David W. Kennedy, ${ }^{1}$ James L. Kreindler, ${ }^{3}$ Robert F. Margolskee, ${ }^{4}$ and Noam A. Cohen ${ }^{1,5}$ \\ 1Department of Otorhinolaryngology, Head and Neck Surgery, University of Pennsylvania, Philadelphia, Pennsylvania, USA. \\ ${ }^{2}$ Division of Neurology and ${ }^{3}$ Department of Pediatrics, Children's Hospital of Philadelphia, Philadelphia, Pennsylvania, USA. ${ }^{4}$ Monell Chemical Senses Center, \\ Philadelphia, Pennsylvania, USA. ${ }^{5}$ Philadelphia Veterans Affairs Medical Center Surgical Services, Philadelphia, Pennsylvania, USA.
}

\begin{abstract}
Bitter taste receptors (T2Rs) in the human airway detect harmful compounds, including secreted bacterial products. Here, using human primary sinonasal air-liquid interface cultures and tissue explants, we determined that activation of a subset of airway T2Rs expressed in nasal solitary chemosensory cells activates a calcium wave that propagates through gap junctions to the surrounding respiratory epithelial cells. The T2Rdependent calcium wave stimulated robust secretion of antimicrobial peptides into the mucus that was capable of killing a variety of respiratory pathogens. Furthermore, sweet taste receptor (T1R2/3) activation suppressed T2R-mediated antimicrobial peptide secretion, suggesting that T1R2/3-mediated inhibition of T2Rs prevents full antimicrobial peptide release during times of relative health. In contrast, during acute bacterial infection, T1R2/3 is likely deactivated in response to bacterial consumption of airway surface liquid glucose, alleviating $\mathrm{T} 2 \mathrm{R}$ inhibition and resulting in antimicrobial peptide secretion. We found that patients with chronic rhinosinusitis have elevated glucose concentrations in their nasal secretions, and other reports have shown that patients with hyperglycemia likewise have elevated nasal glucose levels. These data suggest that increased glucose in respiratory secretions in pathologic states, such as chronic rhinosinusitis or hyperglycemia, promotes tonic activation of T1R2/3 and suppresses T2R-mediated innate defense. Furthermore, targeting T1R2/3-dependent suppression of T2Rs may have therapeutic potential for upper respiratory tract infections.
\end{abstract}

\section{Introduction}

Mucociliary clearance is the primary physical defense against inhaled pathogens, toxins, and particulates in the respiratory system (1) and is complemented by the generation of antimicrobial peptides (AMPs) and radicals that contribute to mucosal innate immunity and maintenance of a clean airway (2). Defining the signaling pathways underlying these processes is critical for understanding and developing therapeutics for respiratory diseases, including chronic rhinosinusitis (CRS), a disease affecting nearly 35 million Americans, with an aggregated cost of $\$ 6$ billion annually, and accounting for 1 in 5 antibiotic prescriptions in adults (3-6).

Bitter taste receptors (T2Rs) (7) are emerging as novel regulators of innate immunity in the respiratory tract (8). Originally identified in type II taste receptor cells of the tongue, T2Rs are thought to protect against the ingestion of toxic compounds, including bacterial products from spoiled foods. T2Rs are G protein-coupled receptors previously shown to be expressed in the cilia of human bronchial (9) and sinonasal epithelial cells (10), in which their activation increases ciliary beat frequency $(\mathrm{CBF})$ and thus mucociliary clearance. Additionally, we previously showed that T2R38, one of the T2Rs expressed in upper respiratory ciliated cells, is stimulated by acyl-homoserine lactones secreted by gram-negative bacteria, resulting in generation of bactericidal amounts of nitric oxide (10). In addition to expression in ciliated cells, T2Rs are also expressed in solitary chemosensory cells (SCCs), which were previously identified in the rodent (11) and,

Conflict of interest: The authors have declared that no conflict of interest exists. Citation for this article: J Clin Invest. 2014;124(3):1393-1405. doi:10.1172/JCI72094. recently, the human $(12,13)$ nose. SCCs also express the T1R2 and T1R3 subunits comprising the human sweet taste receptor (14). Activation of the T2Rs in mouse nasal SCCs stimulates a trigeminal nerve-mediated reduction in respiratory rate (11). However, the function of nasal SCCs in humans and the role of the sweet taste receptor in these cells remain unknown.

T2R-mediated detection of bacteria may be critically important in the nose, which is at the front line of respiratory defense. Thus, the goal of this study was to further understand the role of mammalian taste receptors in upper airway physiology and innate immunity. We demonstrate an important and novel role for T2Rs in human sinonasal epithelial defense. We also present evidence that T2R function is regulated by T1R sweet taste receptor function, further demonstrating the importance of taste signaling to mucosal innate immunity. Moreover, these novel roles for T2Rs and sweet taste receptors in AMP secretion are unique to the human upper airway. While T2Rs activate robust calcium responses in ciliated cells derived from the lower airway or mouse nasal SCCs, they do not appear to regulate antimicrobial peptide secretion. Finally, these results have potentially important clinical implications, because they suggest a novel pathophysiological mechanism that may contribute to upper respiratory infections in patients with CRS and hyperglycemia.

\section{Results}

$T 2 R$ agonists activate localized calcium signals in human sinonasal epithelial cells. In type II taste receptor cells of the tongue, T2Rs signal through elevation of intracellular calcium (15). To look for evidence of SCC T2R activity in human upper airway cells, we performed fluo-4 calcium imaging of primary human sinonasal epi- 
thelial cells (HSECs) isolated from surgical explants and cultured at an air-liquid interface (ALI), thereby recapitulating a polarized, differentiated respiratory epithelium (10). We have previously demonstrated that T2R38 agonists, e.g., PTC, induce global calcium responses in sinonasal epithelial cell cultures, due to expression of T2R38 in the overwhelming ciliated cells (10). In looking to expand on these observations, we examined the effects of other bitter agonists that specifically activate other T2Rs to determine whether we could observe more localized calcium responses, potentially emanating from SCCs. We initially tested the bitter compound denatonium benzoate, which activates 8 different T2Rs but not T2R38 (16) and has previously been used to stimulate mouse nasal SCCs (17). Denatonium benzoate application to the apical/mucosal surface of human sinonasal ALI cultures resulted in dose-dependent elevation of intracellular calcium (Figure 1, $\mathrm{A}$ and $\mathrm{B}$ ) originating from discrete cells that subsequently spread to the surrounding cells (Figure 1C). The global calcium responses we observed previously in response to T2R38 activation (10) were not observed, which was expected because denatonium does not activate T2R38 (16). However, this result also suggests that the ciliated epithelial cells expressing T2R38 do not express denatonium-responsive T2Rs. Denatonium-responsive T2Rs are instead expressed in a different cell type. This is intriguing, as it suggests that denatonium-responsive T2Rs serve a unique and likely novel function from the T2R38 nitric oxide response observed in ciliated cells. The calcium propagation was sensitive to gap junction inhibitors carbenoxolone, $18 \alpha$-glycyrrhetinic acid, and Gap 27 but not to apyrase, which degrades extracellular ATP (Figure 1, $\mathrm{D}$ and $\mathrm{E}$, and Supplemental Figure 1, A-C). This result implicates signal propagation through gap junction communication but not through the release of extracellular purines. Application of denatonium to the basolateral/serosal surface (Supplemental Figure 2A) had no effect, and denatonium stimulation did not alter the magnitude or kinetics of subsequent ATP responses (Supplemental Figure 2, B-D), suggesting that the calcium response was not due to any toxic effects of denatonium but rather activation of apical receptors. Furthermore, denatonium-induced calcium responses were blocked by inhibition of PLC $\beta 2$ (Supplemental Figure 3A), an important downstream component of taste signal transduction (18). The duration of denatonium-induced calcium responses was reduced by either removal of extracellular calcium or application of triphenylphosphine oxide (Supplemental Figure 3B), an inhibitor of the TRPM5 component of taste signaling (19). Additionally, the inositol triphosphate receptor $\left(\mathrm{IP}_{3} \mathrm{R}\right)$ inhibitor xestospongin B significantly inhibited the denatonium-induced calcium response (Supplemental Figure 3C). Repeated stimulation with denatonium induced minimal tachyphylaxis (Supplemental Figure 4), suggesting that these cells recover their signaling capacity (e.g., refill calcium stores) quickly. Together, these initial results strongly suggest that denatonium benzoate activates apical membrane T2R(s), which results in a unique pattern of calcium signaling involving PLC, $\mathrm{IP}_{3} \mathrm{Rs}$, and TRPM5.

The localized denatonium responses observed in sinonasal epithelial cells (upper airway) contrast with those in human bronchial epithelial cells (lower airway), where denatonium responses are more global (ref. 9 and Supplemental Figure 5A). To confirm that the sinonasal cultures reflected the in vivo response, we performed calcium imaging of human sinonasal explants. The resulting denatonium-induced calcium responses also appeared to emanate from distinct points of origin similar to those observed in sinonasal cul- tures (Supplemental Figure 5B). These cultures contain ciliated cells and goblet cells and likely contain SCCs, and our data suggest that they recapitulate the in vivo epithelial physiology. We found that primary enzymatically dissociated bronchial ciliated epithelial cells responded to denatonium (Supplemental Figure 6A), but nasal ciliated epithelial cells did not, despite robust ATP-induced responses (Supplemental Figure 6B). This contrasted with T2R38 agonists, which activate calcium signaling in dissociated nasal ciliated cells (10), suggesting that the denatonium-responsive airway T2R(s) is/are not expressed in sinonasal ciliated cells. In the process of screening bitter compounds, we found that absinthin activated a similar pattern of calcium signaling to that of denatonium and that it originated from the same denatonium-responsive cells (Supplemental Figure 7, A-D). Impalement of the sinonasal denatonium-responsive cells and subsequent injection of Lucifer yellow biocytin in the presence of carbenoxolone confirmed that these cells are nonciliated, unipolar (Supplemental Figure 7E), and morphologically similar to SCCs $(11,12)$ and to cells observed via immunofluorescence for the denatonium and absinthin-responsive T2R47 (Supplemental Figure 7F) using an antibody validated against transfected HEK293 cells (Supplemental Figure 8). Thus, while our prior findings demonstrate that T2R38-mediated innate immunity originates from the ciliated cell (10), the denatonium/ absinthin-responsive cells we observed here were instead nonciliated unipolar cells that are likely human nasal SCCs (12).

Denatonium/absinthin-responsive sinonasal cells express both $T 2 R$ bitter and $T 1 R$ sweet receptors, which negatively regulate the $T 2 R$ response. Mouse nasal SCCs express both T2R bitter and T1R2/3 sweet taste receptors $(14,20-22)$, in contrast to taste cells of the tongue that express either T1Rs or T2Rs. We hypothesized that this may also be the case for human sinonasal cells and asked whether these denatonium-responsive upper airway human cells express T1R2/3 receptors. Like T2R47, we observed that immunofluorescence for the T1R2 subunit of the sweet receptor was localized to solitary nonciliated cells (Supplemental Figure 7G). Costaining of T2R47 with the T1R3 subunit revealed coexpression in the same unipolar cell type (Figure 2), which is likely the same as the SCCs previously described in mice and similar in morphology to the denatonium-responsive cells (Supplemental Figure 7). These cells also appeared to express the type $\mathrm{III} \mathrm{IP}_{3} \mathrm{R}\left(\mathrm{IP}_{3} \mathrm{R} 3\right)$, an intracellular calcium release channel that is expressed in chemosensory cells and important for taste signaling $(23,24)$.

We next sought to examine whether these $\mathrm{T} 1 \mathrm{R} 2 / 3$ receptors expressed in human sinonasal chemosensory cells were functional. T1R2/3 is activated by sugars (e.g., glucose, sucrose) as well as by structurally diverse artificial sweeteners (e.g., sucralose, aspartame) (25), likely due to multiple ligand binding sites (26). We found that denatonium-induced calcium responses were blocked in a dosedependent fashion by apical glucose (Figure 3A), an effect that was reversed by the T1R2/3 antagonist, lactisole (ref. 25 and Figure 3B), but not by inhibitors of glucose transporters (phloretin/phlorizin; Figure 3C). Two other T1R2/3 agonists, sucrose and sucralose, also inhibited denatonium-induced calcium signaling in a T1R2/3dependent manner (that was blocked by the T1R agonists lactisole and amiloride; ref. 27 and Supplemental Figure 9). Together, these data suggest that $\mathrm{T} 1 \mathrm{R} 2 / 3$ receptor activation inhibits denatonium-activated calcium signaling. Neither the T1R2/3 agonists nor lactisole alone had any effect on calcium signaling alone or on ATP-induced calcium kinetics (Supplemental Figure 9). However, when experiments were performed with carbenoxolone to block gap 
A
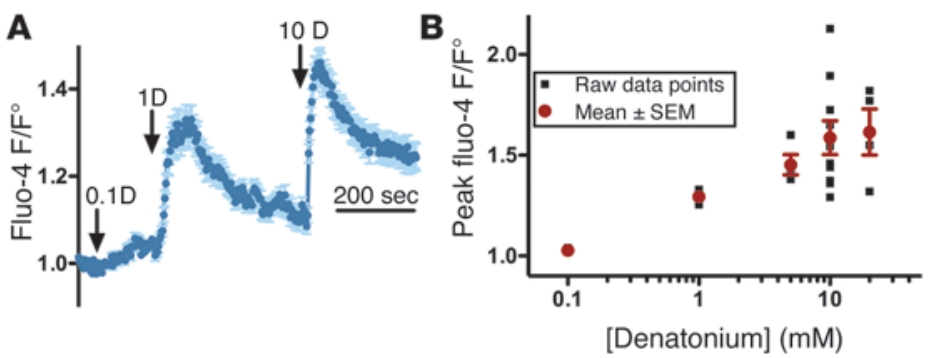

C
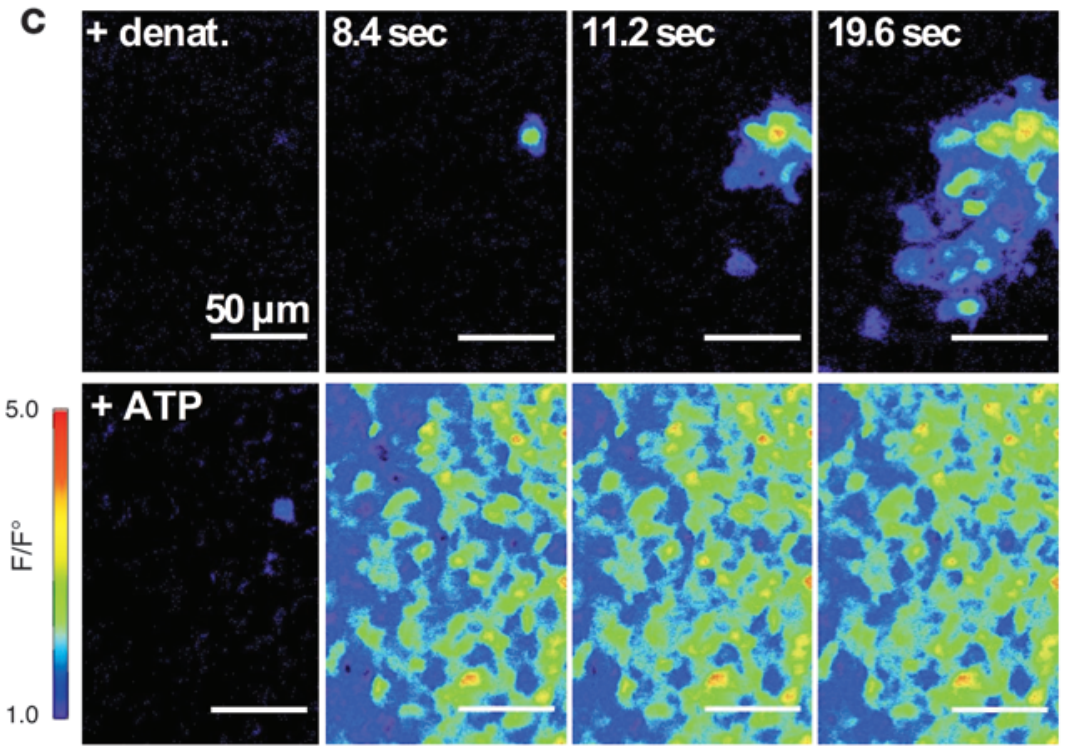

D
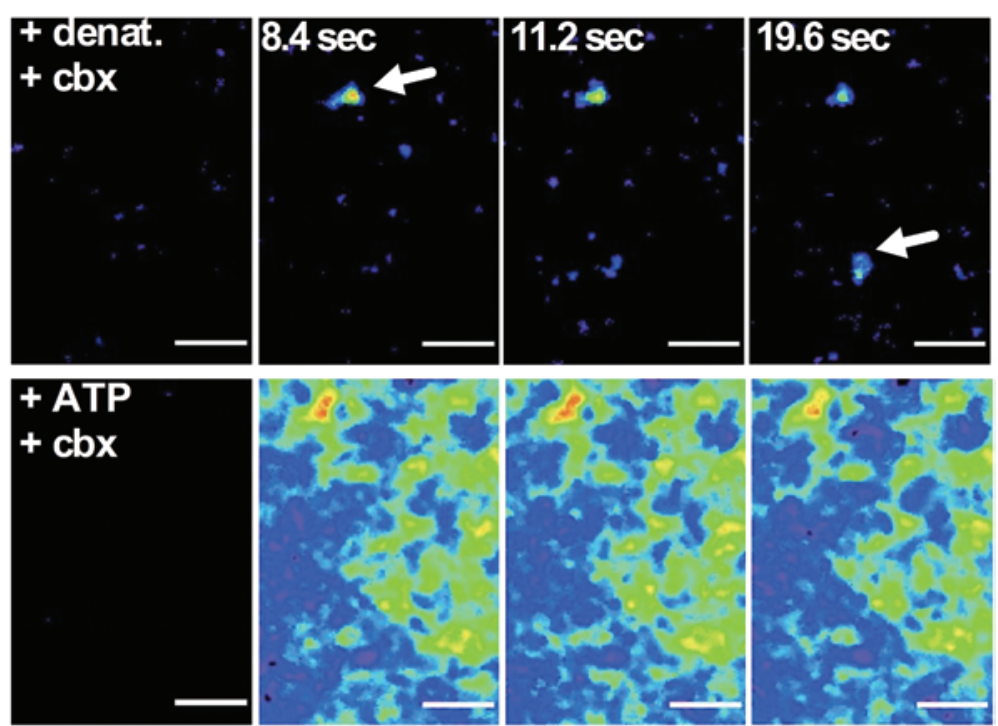

E

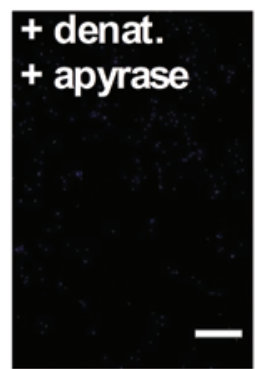

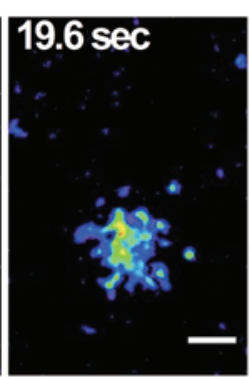

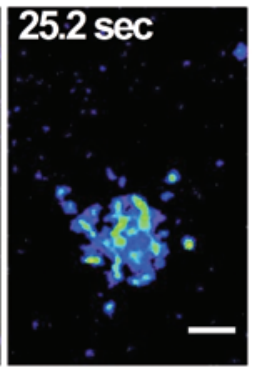

\section{Figure 1}

Calcium responses in sinonasal ALIs during stimulation with denatonium benzoate. (A) Representative trace showing dose-dependent calcium elevations in response to denatonium. $0.1 \mathrm{D}, 0.1 \mathrm{mM}$ denatonium benzoate; $1 \mathrm{D}, 1 \mathrm{mM}$ denatonium benzoate; $10 \mathrm{D}$, $10 \mathrm{mM}$ denatonium benzoate. (B) Dose response plot of data from experiments shown in $\mathbf{A}$. Each red data point is the mean of results from 5 to 12 experiments. (C) Representative images showing propagation of calcium signal from single denatonium-responding cells compared to global calcium mobilization by ATP. denat., denatonium. (D and E) Signal propagation was blocked in the presence of (D) $100 \mu \mathrm{M}$ carbenoxolone (cbx), a gap junction inhibitor, (E) but not apical apyrase. Arrows denote single denatonium-responsive cells. Images shown are representative of 5 to 12 experiments for each condition. Scale bar: $50 \mu \mathrm{m}$.

junctions and isolate the denatonium-responsive cells, glucose inhibited both denatoniumand ATP-induced calcium signals (Figure 3, $\mathrm{D}$ and $\mathrm{E}$ ) within the denatonium-responsive cells but not in cells that did not respond to denatonium. Cells that were not denatonium-responsive exhibited glucose-independent purinergic responses. Thus, these data support the data in Figure 2, showing that discrete sinonasal chemosensory cells express both T2R (bitter) and T1R2/3 (sweet taste) receptors, consistent with SCCs $(14,28)$, and suggest that T1R2/3 activation has direct effects on repressing calcium signaling within these chemosensory cells.

Unlike human sinonasal cultures, we found that denatonium-induced calcium responses in human bronchial epithelial cultures were not inhibited by T1R2/3 agonists (Supplemental Figure 10), suggesting the sweet receptormediated inhibition of the denatonium response may be specific to the upper airway. Together, these results support that the denatoniumresponsive cells in the lower airway are ciliated epithelial cells (9), while the sinonasal denatoniumresponsive cells are nonciliated cells that express both bitter and sweet receptors, strongly suggesting that these cells are sinonasal SCCs (12). Murine nasal SCCs have been more thoroughly characterized than human SCCs $(11,14)$, and thus we also examined murine nasal septal cultures (29) to determine whether the response to denatonium mimicked human sinonasal cultures. We found a similar pattern of denatonium-induced calcium signaling that was likewise inhibited by the T1R2/3 agonists glucose and sucralose (Supplemental Figure 11, A and B). This inhibition was not found in cultures from T1R3 knockout mice (Supplemental Figure 11C). As expected, the denatonium-induced calcium response was absent in gustducin knockout mice and altered (faster decay) in TRPM5 knockout mice but remained intact in cultures derived from 

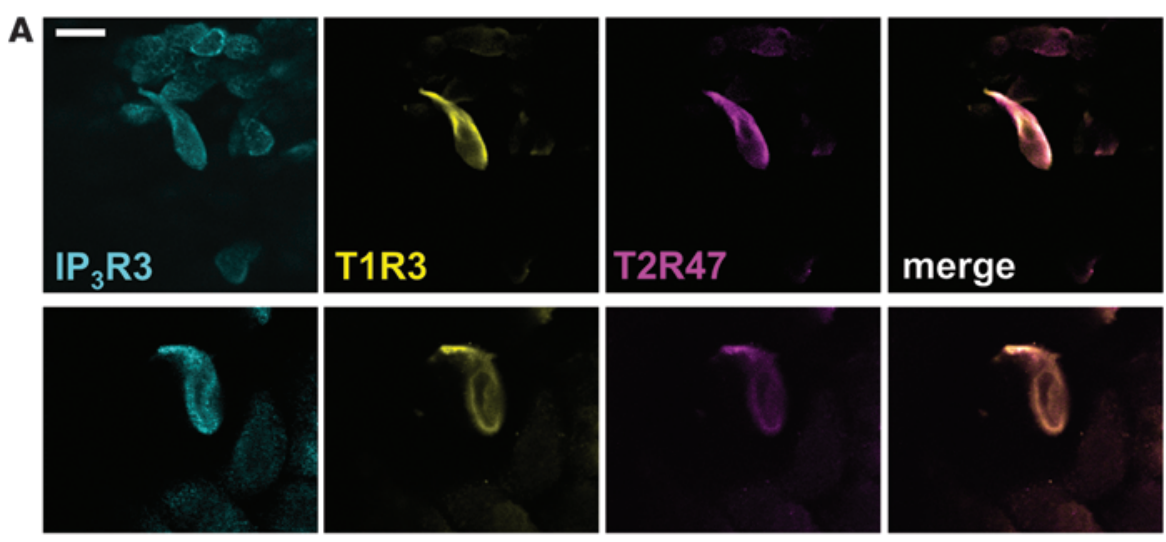

B + T2R47 blocking peptide
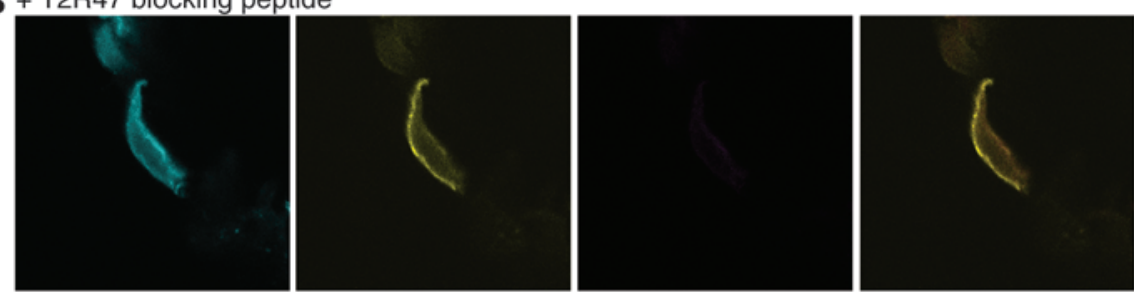

\section{Figure 2}

Human sinonasal chemosensory cells express both bitter and sweet taste receptors as well as $I_{3} R 3$. (A) Cells matching the morphology of SCCs exhibited immunofluorescence for both T2R47 and T1R3. These cells also appeared to express $I P_{3} R 3$, though $I P_{3} R 3$ expression appeared to be more widely distributed than that of T2R47 or T1R3 receptors. Merge shows T1R3 and T2R47 signals. (B) T2R47 staining was reduced when T2R47 antibody was preincubated with antigenic blocking peptide ( 2 hours) before staining. Scale bar: $10 \mu \mathrm{m}$.
Myd88 knockout mice, suggesting that it is independent of TLR signaling (Supplemental Figure 11, E-I) and further supporting this response as specific to taste signaling.

Denatonium/absinthin-stimulated $T 2 R$ activation does not stimulate $C B F$ in HSECs. Prior work suggested that denatonium stimulates calcium-dependent increases in CBF in human bronchial cells (9), and, thus, we sought to determine whether this is the case in sinonasal cells. Interestingly, we found that application of denatonium benzoate slowed CBF in sinonasal cells (Supplemental Figure 12, A-D). We hypothesize that this effect is from intracellular acidification secondary to permeation of benzoic acid. When intracellular $\mathrm{pH}$ buffering capacity was increased by addition of $\mathrm{CO}_{2} / \mathrm{HCO}_{3}$ to the basolateral media (30), the CBF decrease was blunted, but still no increase was observed (Supplemental Figure 12, F-I). Direct imaging of intracellular $\mathrm{pH}$ suggested that $10 \mathrm{mM}$ denatonium benzoate and $10 \mathrm{mM} \mathrm{Na}$ benzoate caused a similar level of intracellular acidification. In agreement with this hypothesis, neither denatonium saccharide, which lacks the benzoic acid moiety, nor the other T2R agonists absinthin, parthenolide, or amarogentin caused a decrease in CBF (Supplemental Figure 12, $\mathrm{J}$ and $\mathrm{K}$ ). These agonists also failed to cause any CBF increase. This agrees with our data suggesting that sinonasal ciliated epithelial cells do not express functional denatonium- or absinthinresponsive T2Rs. It is not likely that the SCC-activated gap junction-propagated calcium response is properly localized to elevate $\mathrm{CBF}$, a phenomenon similar to what we have previously observed when sinonasal epithelial cells are stimulated with histamine, an agonist that elevates calcium and stimulates fluid secretion but nonetheless has no effect on CBF (31). Additionally, the presence of sugar in the airway surface liquid (ASL) had no significant effect on CBF (Supplemental Figure 12). These data suggest the denatonium/absinthin-responsive cells have a different role in the human upper airway compared with that in the lower airway. Because we have shown that T2R38 detects gram-negative bacterial quorumsensing molecules and activates innate immunity responses (10), we hypothesized that the denatonium- and absinthin-responsive T2Rs likewise serve a role in innate immunity and thus began to examine other sinonasal epithelial innate immunity responses.

Denatonium/absinthin-stimulated T2R activation results in potent antimicrobial secretion that is inhibited by ASL glucose. Although denatonium benzoate does not stimulate human sinonasal ALIs to produce bactericidal nitric oxide or reactive nitrogen species (10) or to secrete cytokines (Supplemental Figure 13), we found that denatonium does induce a bactericidal response. When we stimulated the apical side of human sinonasal ALI cultures with PBS in the presence or absence of $10 \mathrm{mM}$ denatonium benzoate for 30 minutes at $37^{\circ} \mathrm{C}$ and then collected the ASL and mixed it with Psendomonas aeruginosa, we observed that ASL from denatonium-stimulated cultures was potently bactericidal compared with ASL from cultures exposed to PBS alone, which had minimal effect on bacterial viability (Figure 4, A and B). The solutions used in these experiments had no cell-independent effects on bacterial growth when tested alone, and there was no antibacterial activity when denatonium was added to PBS ASL after removal from the cells (Supplemental Figure 14, A-C). Denatonium-induced antimicrobial ASL activity against Pseudomonas was inhibited by calcium chelation or inhibition of PLC $\beta 2$ or gap junction function (Figure 4C). Together, these data suggest that this is a receptor- and calcium-dependent response and that the propagation of the T2R calcium signal to the surrounding cells is essential for antimicrobial secretion. Interestingly, inhibition of TRPM5 had no effect on bacterial killing (Figure 4C), suggesting that the observed antibacterial effects are dependent on intracellular calcium release. In agreement with the calcium results presented above, the antimicrobial effects of denatonium were inhibited by glucose in a lactisole-reversible manner (Figure 4C), supporting a role for T1R2/3 in inhibition of sinonasal denatonium T2R signaling. The exact mechanism(s) of the glucose-mediated inhibition are unknown but likely are dependent on cAMP and protein kinase signaling (Supplemental Figure 14, $\mathrm{D}$ and $\mathrm{E}$ ), which suggests that signaling architecture of SCCs may 

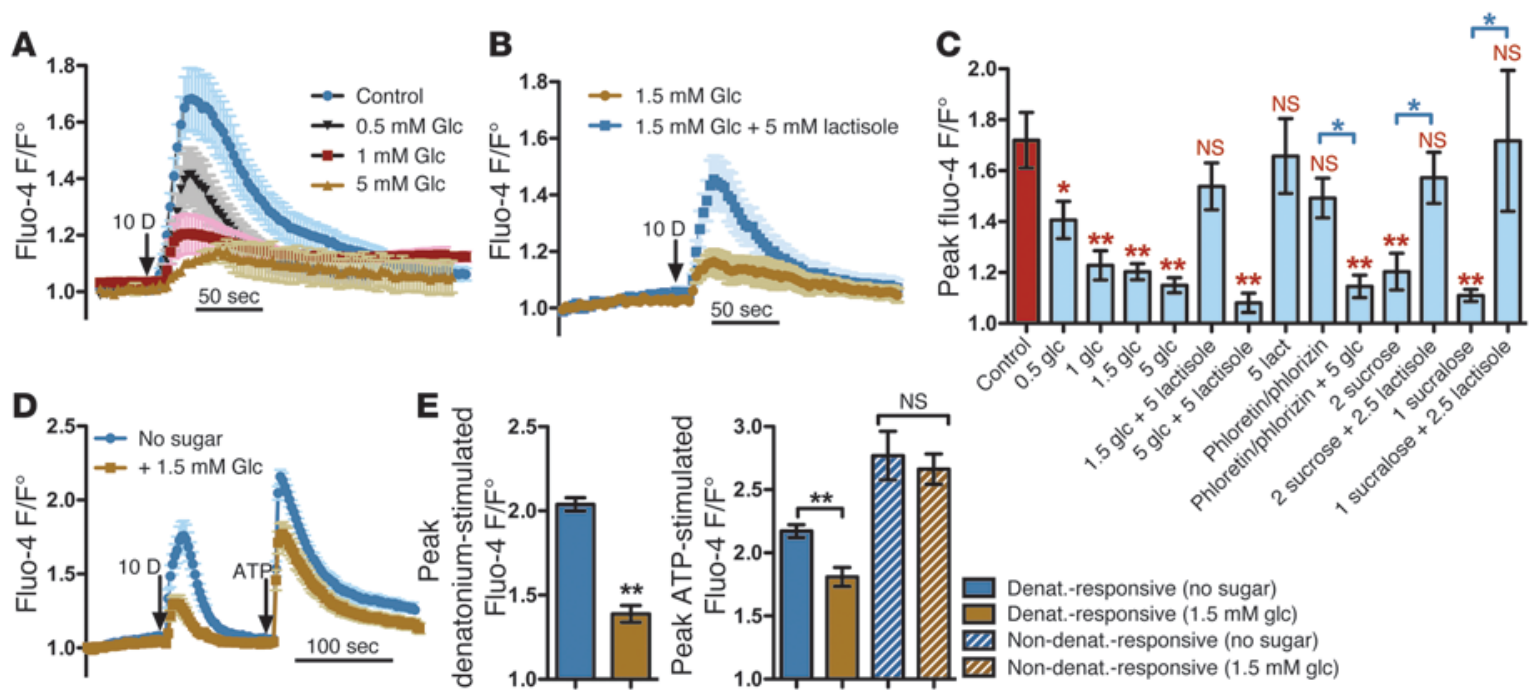

Figure 3

Sweet receptor activation inhibits T2R-activated calcium signaling. (A) Dose-dependent glucose (Glc) inhibition of the calcium response to $10 \mathrm{mM}$ denatonium benzoate (mean \pm SEM; $n=5-9$ experiments using cultures from at least 3 patients each). (B) Inhibition is reversed by lactisole (mean \pm SEM; $n=5-7$ cultures from at least 3 patients each). (C) Peak calcium responses (mean \pm SEM; 6-12 cultures from at least 3 patients each) and inhibition by glucose, sucrose, and sucralose (but not phloretin/phlorizin) as well as reversal of inhibition by lactisole. Red asterisks denote significance versus control (10 mM denatonium stimulation alone) determined by 1-way ANOVA with Dunnett's post-test. Blue asterisks denote significance between bracketed bars determined by 1-way ANOVA with Bonferroni post-test. Experiments in A-C were performed without carbenoxolone. (D) In the presence of carbenoxolone, single denatonium-responsive cells exhibited glucose inhibition of both denatonium- and ATP-induced calcium signals (mean \pm SEM; 18-20 cells from 3 patients for each condition). (E) Peak denatonium-induced calcium response (mean \pm SEM) in the presence of carbenoxolone with or without glucose (significance determined by Student's $t$ test) from experiments in D. ATP response (mean \pm SEM) in denatonium-responsive (solid bars) and nonresponsive (hatched bars) cells (significance determined by 1 -way ANOVA with Bonferroni post-test). ${ }^{*} P<0.05$; ${ }^{* \star} P<0.01$.

follow some models proposed for gustatory type II cell signaling, where T2Rs lower intracellular cyclic nucleotide levels (typically through activation of the G $\alpha$ protein gustducin) and T1R receptors increase cAMP levels (32). Notably, these cells expressed IP 3 R3 (Supplemental Figure 7), like other chemosensory cells (33). IP 3 R3 phosphorylation by PKA has been suggested to inhibit calcium signaling in some cells, including pancreatic acinar cells $(34,35)$. A similar mechanism may be operating here.

Similar to denatonium, absinthin-stimulated ASL was bactericidal (Figure 4D), while ASL from cultures stimulated with sodium benzoate, other T2R agonists, ATP, and TLR agonists was not (Figure 3D and Supplemental Figure 15). These results follow the calcium data presented above and suggest that this response is unique to taste signaling mediated by a subset of T2Rs. Denatonium-stimulated ASL also effectively killed methicillin-resistant Staphylococcus aureus (MRSA), Klebsiella pneumonia, and Staphylococcus epidermis, suggesting that the secretions are broad-spectrum antimicrobials (Figure 4E).

These data strongly suggest that T2Rs have novel roles in sinonasal innate immunity beyond the role we previously demonstrated for T2R38 (10), strengthening the hypothesis that airway T2Rs represent a novel arm of innate immunity, functioning on a much faster time span than TLRs and Nod-like receptors (36), and may be important therapeutic targets for upper respiratory infections. Thus, we examined the denatonium-stimulated antimicrobial secretions in more detail. The secretion of bactericidal activity occurred within 10 minutes of denatonium stimulation, while bacterial killing (a reduction in the number of CFUs) occurred over a time course of approximately 2 hours of exposure to the secreted antimicrobials (Figure 5, A and B). Notably, after stimulation and secretion of bactericidal compounds, the ALI cultures exhibited a refractory period before they could mount a second antibacterial secretion response, as ASL from cultures stimulated the day after a prior stimulation (1-day recovery) did not kill Psendomonas. Rather, cultures required a minimum of 3 days of recovery to restore bactericidal activity in the ASL (Figure 5C). This suggested that protein synthesis is required and that the secreted factors may be AMPs. SDS-PAGE, with subsequent Coomassie blue staining, revealed a large amount of low-molecularweight protein(s) in denatonium-stimulated ASL (Figure 6A). When denatonium-stimulated secretions were fractionated by size, antipseudomonal activity was retained in the $<30-\mathrm{kDa}$ fraction (Figure 6B). Dialysis of denatonium-stimulated secretions against $>2,000$-fold volume of PBS revealed that antibacterial activity was retained with a low $(3-\mathrm{kDa})$ molecular weight cutoff (MWCO) but lost with larger MWCOs (Figure 6B). We hypothesized that bactericidal activity was mediated by low-molecular-weight AMPs known to be secreted by the sinonasal epithelium (37), including $\beta$-defensins. Supporting this, we found that $\mathrm{NaCl}$ inhibited the bactericidal activity in a dose-dependent fashion. Antibacterial activity was reduced with increasing $[\mathrm{NaCl}]$ (Figure 6C) in a manner independent of any effects of $\mathrm{NaCl}$ on secretion (described in the Methods). Increasing ionic strength prevents AMP function $(38,39)$, likely by shielding charge and preventing AMP interactions necessary for antibacterial effects. ELISAs revealed that denatonium and absinthin, as well as the T2R ago- 
A

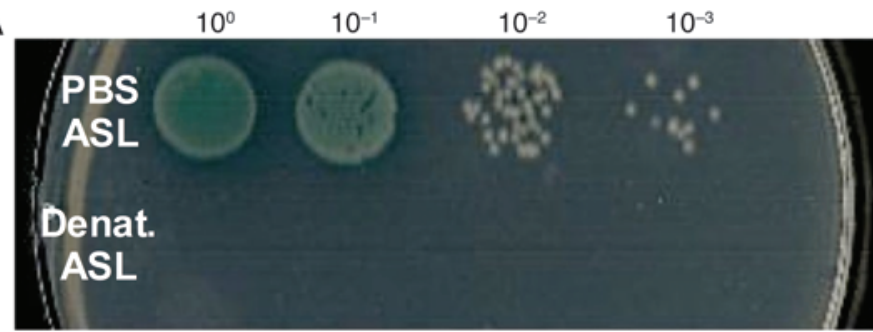

B
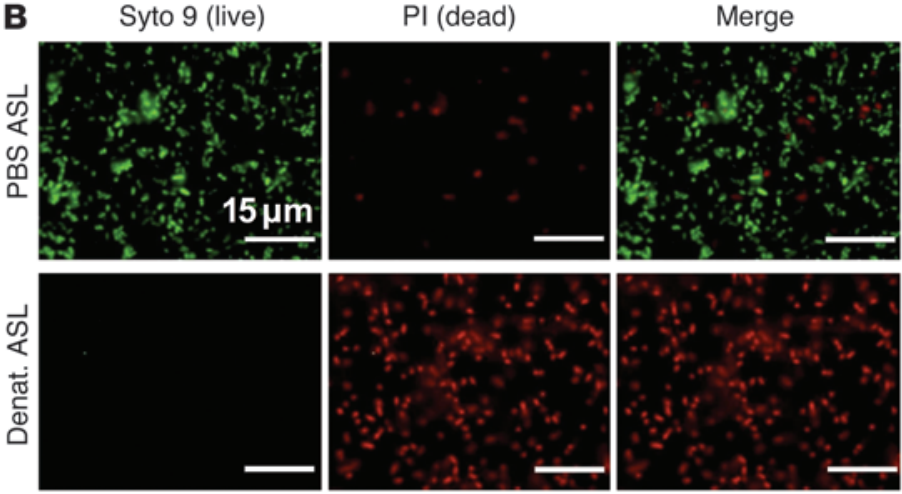

C

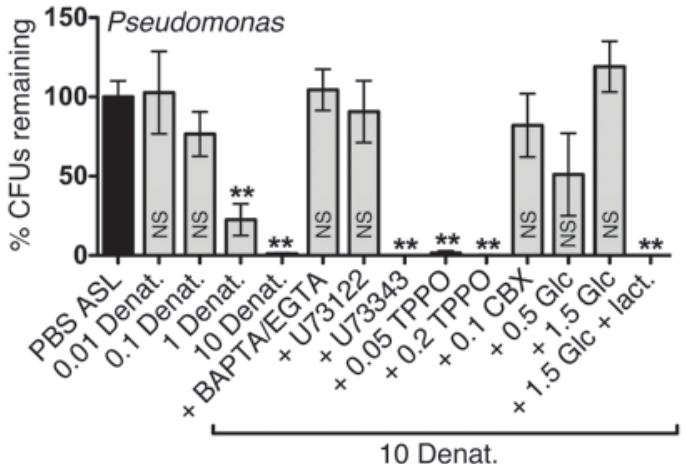

10 Denat.

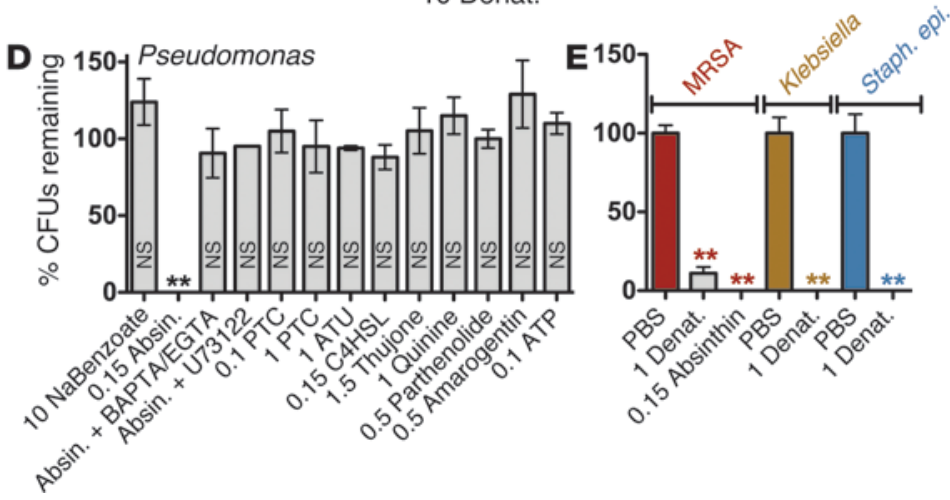

\section{Figure 4}

Denatonium/absinthin-sensitive T2Rs activate sinonasal epithelial cells to secrete compounds with potent and broad-spectrum antimicrobial activity. (A) Bacterial CFUs when Pseudomonas were mixed with ASL from ALI cultures stimulated with PBS or $10 \mathrm{mM}$ denatonium. (B) Confirmation of bacterial kill by live-dead staining of planktonic Pseudomonas. Syto 9 and propidium iodide $(\mathrm{PI})$ indicate live and permeabilized (dead) bacteria, respectively (10). The images are representative of 5 experiments using ASL from 5 patients. Scale bar: $15 \mu \mathrm{m}$. (C and D) Percentage Pseudomonas CFUs remaining (mean \pm SEM) when bacteria were mixed with ASL from ALIs stimulated under indicated conditions. Secretion of antimicrobial products was inhibited by inhibition of calcium signaling (BAPTA/EGTA), gap junction communication (carbenoxolone, CBX), or PLC 32 (U73122) as well as by glucose. Glucose inhibition was reversed by lactisole (lact.). Inhibition was not observed with the TRPM5 inhibitor triphenylphosphine oxide (TPPO) (mean \pm SEM; data from 12-66 cultures from at least 3 different patients for each condition). Concentrations shown are in $\mathrm{mM}$. ${ }^{* *} P<0.01$, vs. control (PBS) determined by 1 -way ANOVA with Dunnett's post-test. (E) Activity of ASL secretions against other bacteria species (mean \pm SEM; $n=6-16$ cultures from at least 3 different patients for each condition). ${ }^{* *} P<0.01$, each species vs. control (PBS) determined by 1-way ANOVA with Bonferroni post-test. nists parthenolide and amarogentin, stimulated secretion of $\beta$-defensins 1 and 2 that was blocked by inhibition of calcium signaling, gap junction inhibition, PLC $\beta 2$ inhibition, or glucose (Figure 6, $\mathrm{D}$ and $\mathrm{E})$. Parthenolide and amarogentin stimulated approximately one-third the amount of $\beta$-defensin observed with denatonium simulation, likely explaining why they had no effect in our antimicrobial assay (Figure 4D). Our preliminary studies showed that a dilution of as little as 1:5 of the denatonium-stimulated ASL from this assay was enough to eliminate the antimicrobial activ- ity; this is likely due to the limitations brought about by the need to use an experimental fluid volume on the apical surface of our cultures that is much higher than the physiological 7- to $10-\mu \mathrm{m}$ high ASL layer.

These data suggest that a subset of T2Rs in human sinonasal chemosensory cells, likely SCCs, stimulate secretion of AMPs from neighboring epithelial cells, constituting a novel trigger of mucosal innate immunity. In the human lung, $\beta$-defensin 2 is expressed in epithelial cells and also submucosal glands (39); 

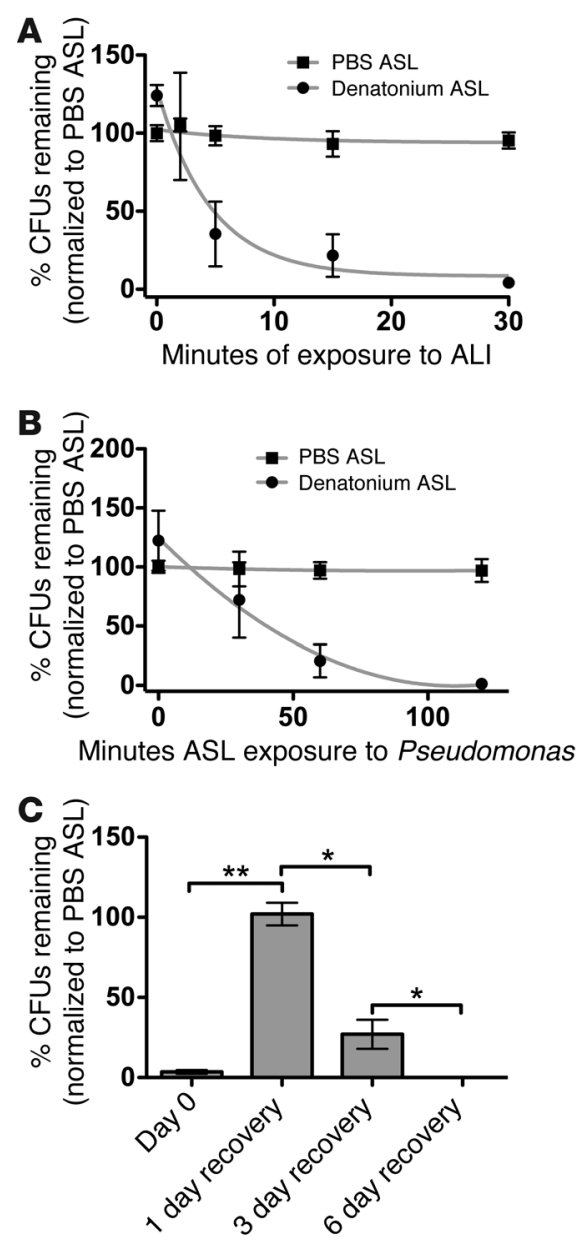

\section{Figure 5}

Kinetics of the denatonium-induced antimicrobial response. (A) ALI cultures were exposed to denatonium for various times, and ASLs were then incubated to Pseudomonas. Resulting CFUs remaining (normalized to PBS) are shown (mean \pm SEM; $n=6-8$ experiments from 6 to 8 cultures from at least 3 patients for each condition). Decreased CFU count equals a reduced number of viable bacteria. Human sinonasal ALI cultures secreted antimicrobials over the course of 5 to 10 minutes. (B) ALI cultures were stimulated with denatonium for 30 minutes, and ASL was mixed with Pseudomonas for varying periods of time before dilution and spotting on LB plates. Resulting CFUs remaining (normalized to PBS) are shown (mean \pm SEM; $n=$ ALI cultures from 4 patients for each condition). Maximal killing was achieved only after $>90$ minutes of exposure of bacteria to ASL. (C) Cultures were stimulated with $10 \mathrm{mM}$ denatonium benzoate and then either stimulated the next day (1-day recover) or given 3 or 6 days to recover. A second stimulation of maximal killing of Pseudomonas was achieved only after cultures were allowed to recover for $>3$ days. CFUs remaining (mean \pm SEM) are shown $(n=4-12$ ALI cultures from at least 4 patients for each condition). ${ }^{*} P<0.05$; ${ }^{* *} P<0.01$, determined via 1-way ANOVA with Bonferroni post-test.

however, most studies of $\beta$-defensin expression in the upper airway have focused on mRNA expression or $\beta$-defensin protein concentrations in nasal secretions $(40,41)$ rather than cellular localization or levels of $\beta$-defensin protein. Confirming the hypothesis that denatonium-responsive $\mathrm{T} 2 \mathrm{R}$ s are responsible for regulating rapid AMP secretion, we noted that human sinonasal ALIs exhibited apical $\beta$-defensin immunofluorescence that was markedly reduced 30 minutes after denatonium treatment (Supplemental Figure 16). This was confirmed by ELISA of cell lysates, revealing that denatonium decreased intracellular $\beta$-defensin by approximately $80 \%$ to $90 \%$; several days after stimulation were required to return to baseline levels (Figure $6 \mathrm{~F}$ ).

TLRs are also known to regulate production and secretion of $\beta$-defensin 2 (but not $\beta$-defensin 1 , which is thought to be constitutively expressed) $(40,41)$, but more often TLR activation seems to result in slower but sustained upregulation of $\beta$-defensin 2 mRNA and protein, not the rapid release we saw here with T2R agonists. In support of this, we found that stimulation with a cocktail of TLR agonists caused an increase in intracellular levels of $\beta$-defensin 2 (but not $\beta$-defensin 1 ) after approximately 12 hours stimulation (Supplemental Figure 17). Additionally, 24-hour stimulation with a TLR cocktail resulted in an increased level of denatonium-stimulated $\beta$-defensin 2 release (Supplemental Figure 17), suggesting that these two pathways are complementary. While both result in innate defense through AMP production, T2Rs appear to be more important for immediate release of cellular contents, while TLRs appear to be important for increasing and sustaining production of inducible AMPs.

Importantly, murine nasal and human bronchial ALIs did not exhibit denatonium-induced antimicrobial/ $\beta$-defensin secretion (Supplemental Figure 18), suggesting the response is specific to the human upper airway. To test whether $\beta$-defensin secretion was necessary for the sinonasal antimicrobial response to T2R agonists, antibodies to $\beta$-defensins 1,2 , and 3 were added to denatoniumstimulated ASL before mixture with Pseudomonas. Bactericidal effects against both Pseudomonas and MRSA were significantly inhibited compared with denatonium ASL that was preincubated without antibodies (Figure 6G). Likewise, when the same antibodies were used with protein A-sepharose to immunoprecipitate $\beta$-defensins (overnight incubation at $4^{\circ} \mathrm{C}$ ), bactericidal effects were blocked compared with denatonium-stimulated secretions that were incubated with protein A-sepharose only (Figure 6G). However, the inhibition of bactericidal activity was not complete, suggesting that other non- $\beta$-defensin antimicrobial components were also secreted. Airway epithelial cells secrete many other antimicrobials, including lysozyme, lactoferrin, and LL-37 (42-45). While our data do not rule out the effects of any of these molecules, they do support that hypothesis that $\beta$-defensins play a major role in the human nasal SCC innate immunity response.

We confirmed the in vivo relevance of these observations by testing whether denatonium could activate secretion of $\beta$-defensins from intact pieces of excised human turbinate mucosa. We found that turbinate incubated with $10 \mathrm{mM}$ denatonium benzoate secreted significantly increased amounts of $\beta$-defensins 1 and 2 that were markedly reduced in the presence of $1.5 \mathrm{mM}$ glucose (Figure 6H). This result supports that the effects we observed in ALI cultures were relevant for human sinonasal physiology. To begin to hypothesize about the physiological role of this effect, we asked whether nasal ASL glucose levels were altered in a common upper respiratory disease, CRS.

Patients with CRS exhibit elevated glucose concentrations in nasal secretions. Since patients with CRS often have recurrent sinonasal infections, we measured glucose in nasal secretions from patients with CRS and control patients and found that patients with CRS (with or without polyps) had elevated glucose con- 

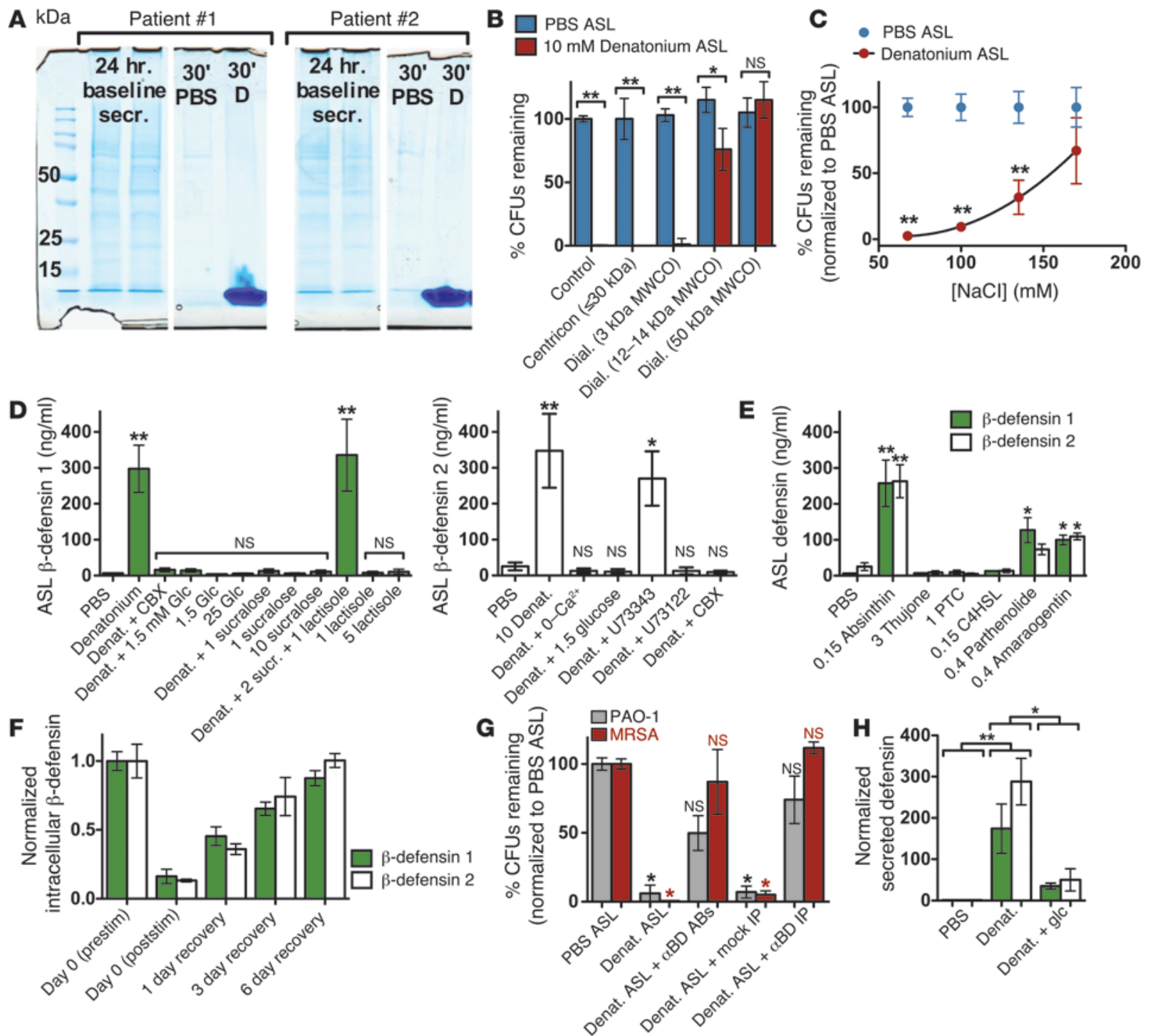

Figure 6

T2R agonists stimulate secretion of $\beta$-defensins from HSECs. (A) Coomassie blue-stained gel showing a low-molecular-weight band in denatonium-stimulated ASL (representative of 3 gels; 6 patients). (B) Denatonium-stimulated secretions were run through a Centricon filter (30-kDa MWCO); antimicrobial activity was retained in the lower molecular weight fraction. Antibacterial activity was retained when ASL from denatonium-treated cultures was dialyzed against 3-kDa MWCO, was reduced with a 12-kDa MWCO, and was fully lost with a 50-kDa MWCO $(n=3-5 \mathrm{ALIs}$ from at least 3 patients each). (C) Antimicrobial function was inhibited by increasing $[\mathrm{NaCl}](n=7$ patients) (significance vs. PBS ASL with same $[\mathrm{NaCl}])$. ( $\mathbf{D}$ and $\mathbf{E}) \mathrm{T} 2 \mathrm{R}$ agonists denatonium, absinthin, parthenolide, and amarogentin stimulate secretion of $\beta$-defensins 1 (green) and 2 (white) $\left(n=5-25\right.$ cultures from $>3$ patients each). ${ }^{\star} P<0.05 ;{ }^{* \star} P<0.01$, compared with PBS ASL, by 1 -way ANOVA with Dunnett's post-test. (F) Intracellular $\beta$-defensin decreased after 30-minute stimulation with $10 \mathrm{mM}$ denatonium and returned to baseline levels by 6 days $(n=5$ cultures from 5 patients each). (G) Bactericidal activity of denatonium-stimulated ASL was blocked by antibodies to $\beta$-defensins (30-minute preincubation) or immunodepletion of $\beta$-defensins ( $n=5$ cultures from 5 patients each). (B, $\mathbf{C}$, and $\mathbf{G}){ }^{*} P<0.05$, ${ }^{*} P<0.01$, determined by 1 -way ANOVA with Bonferroni post-test. $(\mathbf{H})$ Denatonium $(10 \mathrm{mM})$ stimulated $\beta$-defensin 1 and 2 secretion by ex vivo human turbinate that was significantly inhibited by $1.5 \mathrm{mM}$ glucose. Defensin concentration normalized per mg of wet-weight tissue. ${ }^{\star} P<0.05,{ }^{\star \star} P<0.01$. All data are mean \pm SEM.

centrations that were sufficiently high to inhibit antimicrobial secretion in vitro (Figure 7A). A current model for ASL glucose homeostasis suggests that some glucose tonically leaks through the airway epithelium and the low glucose concentrations of normal ASL are kept low by uptake via apical glucose transporters, including GLUT transporters (46-49). Increased ASL glucose has been linked to increased risk of MRSA respiratory infections in some patients (50). Elevated blood glucose (hyperglycemia) alone can result in elevated ASL glucose $(49,51)$, but none of these patients with CRS were known diabetics, and there was no direct correlation between nasal glucose and blood glucose levels (Figure 7B), indicating that elevated glucose concentration in the nasal secretions of these patients is not due to increased serum glucose. The higher nasal glucose is also not likely the result of a genetic difference in epithelial glucose permeability or transport, as ALI cultures derived from 


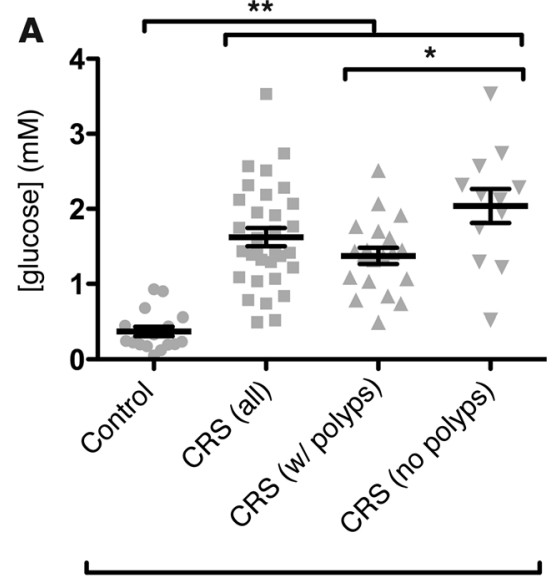

Patient nasal secretions
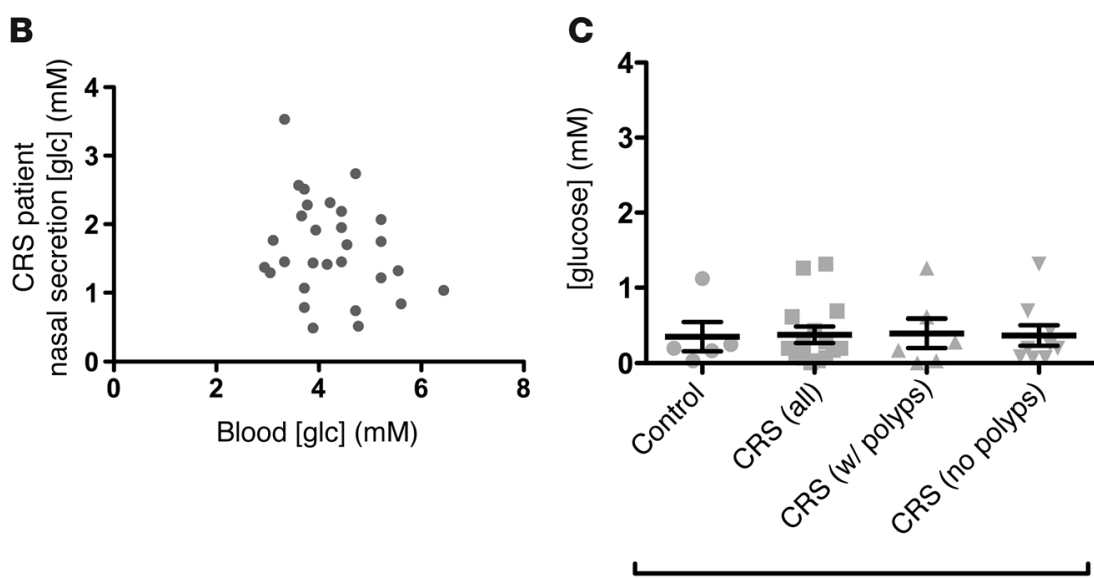

ASL from ALI cultures

Figure 7

Glucose concentrations are elevated in the nasal secretions of patients with CRS. (A) Glucose was measured in nasal secretions from patients with CRS and control individuals. Glucose was $0.37 \pm 0.06 \mathrm{mM}$ vs. $1.63 \pm 0.12 \mathrm{mM}$ in control patients $(n=17)$ and patients with CRS $(n=32)$, respectively. A significant difference was observed between patients with CRS with $(n=20)$ and without $(n=12)$ polyps $(1.4 \pm 0.1 \mathrm{mM}$ vs. $2.0 \pm 0.2 \mathrm{mM}$, respectively), but nonetheless both groups had significantly higher glucose than control patients (mean $\pm \mathrm{SEM}$ ). ${ }^{\star} P<0.05$, ${ }^{\star \star} P<0.01$, determined via 1-way ANOVA with Tukey-Kramer post-test. (B) In patients with CRS, there was no correlation between blood and nasal secretion glucose concentrations ( $n=32$ patients for which nasal and blood glucose values could be obtained). Additionally, none of the patients with CRS used in this study had a prior diagnosis of hyperglycemia, prediabetes, or diabetes. Previous studies have shown that hyperglycemia is correlated with increased nasal secretion glucose $(51,74,75)$. However, these data suggest that the patients with CRS in this study exhibited higher nasal glucose levels due to another mechanism. (C) Glucose was measured in ASL from ALI cultures, with no difference observed among the populations (mean $\pm \mathrm{SEM} ; n=1$ culture each from 5 control patients and 15 patients with CRS, including 6 with polyps and 9 without polyps). No significant differences were detected by 1-way ANOVA.

control patients and patients with CRS had similar low ASL glucose concentrations when grown in identical media (Figure 7C). Instead, the higher glucose concentrations in nasal secretions from patients with CRS likely derives from the sera via epithelial transudate/exudate leaking through the epithelial barrier due to chronic infection/inflammation and epithelial damage that varies with individual patient disease (46). Supporting this, proinflammatory mediators have been shown in vitro to increase paracellular glucose flux in human bronchial cell monolayers (52) and disrupt tight junctions in human sinonasal epithelial cultures $(53,54)$.

We hypothesized that, during infection, bacterial depletion of normal ASL glucose $(\sim 0.5 \mathrm{mM})$ may play an important role in the activation of T2R-mediated AMP secretion. This mechanism may be disrupted in individuals with elevated glucose concentrations $(\sim 1.5 \mathrm{mM})$, in whom it would take longer for bacteria to deplete the glucose, with the possibility of no depletion at all if the glucose leak is significant enough. We tested the ability of Pseudomonas and MRSA to reduce the glucose concentration of an ASL-like fluid and found that these bacteria rapidly consumed glucose (Supplemental Figure 19). However, as expected, it took much longer to consume the glucose concentration of a solution containing $1.5 \mathrm{mM}$ glucose than $0.5 \mathrm{mM}$ glucose. In patients with CRS with elevated glucose levels, this effect may likely give bacteria a longer time period in which to colonize or infect the airways before the activation of any T2R-mediated AMP release.

\section{Discussion}

The work presented in this study strongly complements our prior work (10) and supports the hypothesis that airway taste receptors are a novel arm of human respiratory innate immunity. Genetic variations in these receptors and related pathways may explain an individual's susceptibility to certain respiratory pathogens (10). Furthermore, these receptors and their associated signaling pathways constitute a novel class of therapeutic targets to treat respiratory infections. Taken together, our data identify novel, antagonistic roles of T2R bitter and T1R sweet taste receptors in the human nose, which may have profound implications for upper respiratory diseases, such as CRS and respiratory manifestations of hyperglycemia. The mechanisms of T2R and T1R2/3 interaction remain to be determined in more detail, but our data suggest that $\mathrm{T} 2 \mathrm{R}$ and $\mathrm{T} 1 \mathrm{R} 2 / 3$ receptors are coupled to different signaling pathways. The pharmacological inhibition profile and knockout mouse data suggest that T2R is coupled to the canonical taste signaling pathway, including $\alpha$-gustducin, TRPM5. However, because high concentrations of T1R2/3 agonists do not evoke the same calcium response, T1R2/3 is likely not coupled to $\alpha$-gustducin, but rather to an alternate $G$ protein that remains to be identified. Endogenous T1R receptor expression in adipocytes that do not express gustducin has recently been shown to elevate intracellular cAMP levels through Gas (55), so precedent exists for T1R receptor coupling to $G$ proteins other than gustducin. Our data suggest that T1R2/3 may be linked to cAMP generation and PKA activity, which may inhibit $\mathrm{IP}_{3} \mathrm{R} 3$ activity $(34,35)$ and, thus, reduce calcium signaling in these chemosensory cells.

The role of the T1R2/3 receptor demonstrated here, namely inhibition of T2R-dependent calcium signaling and AMP secretion, is novel. What might be the physiological consequences of human SCCs expressing both bitter and sweet receptors in the same cell? ASL glucose is kept at low levels in healthy individu- 
A

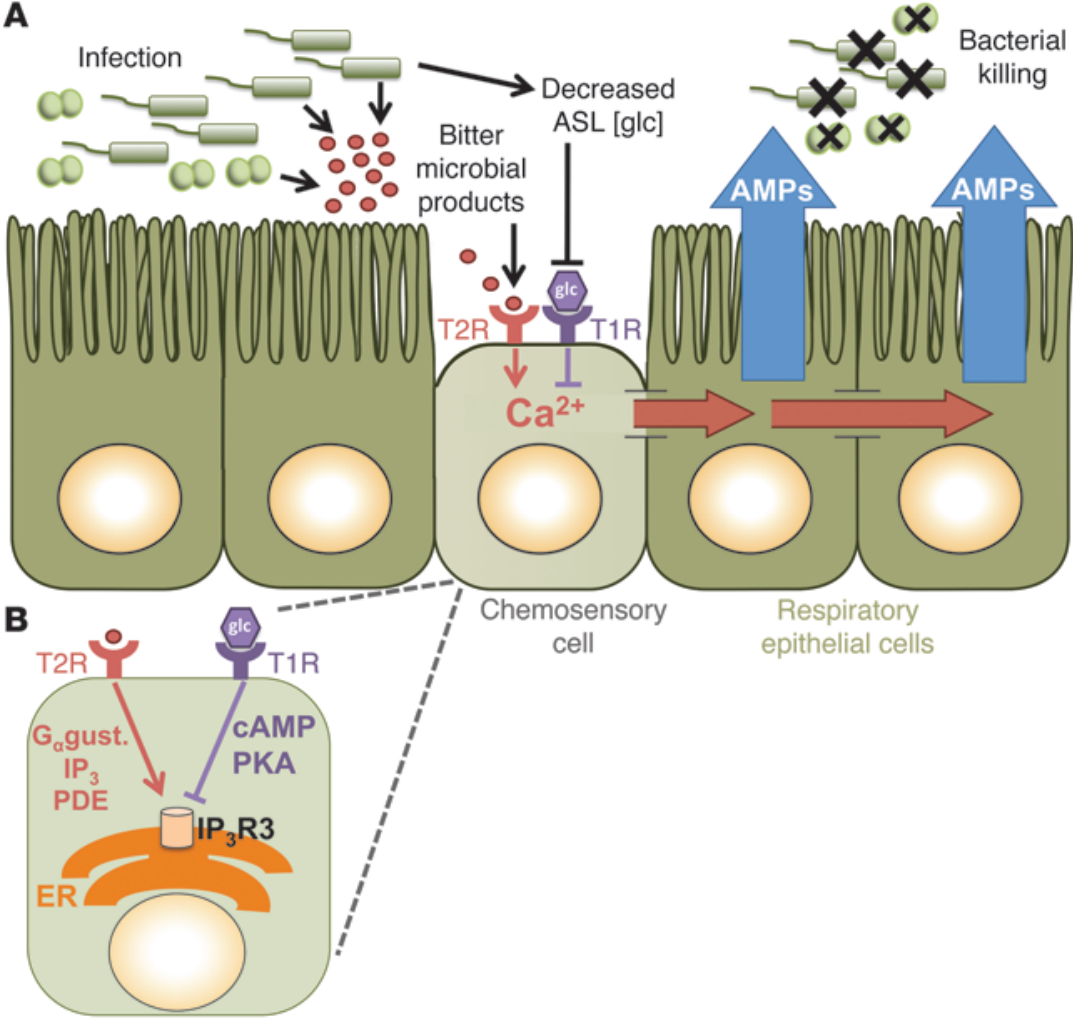

\section{Figure 8}

Proposed model of T2R bitter receptor- and T1R sweet receptor-based regulation of AMP secretion in the human nose. (A) From left to right, bitter chemicals released by microbes during infection activate T2Rs in the sinonasal epithelium (10), likely including those expressed in nonciliated chemosensory epithelial cells, likely the SCCs previously described (11-14). This results in a calcium response that propagates to the surrounding epithelial cells, causing secretion of multiple AMPs, including $\beta$-defensins 1 and 2 , that are capable of direct bacterial killing. Glucose in the ASL normally governs the T2R-mediated response through T1R2/3 activation. However, during acute infections, bacteria may consume glucose (Supplemental Figure 18) and decrease the ASL glucose concentration, which relieves the T1R2/3-mediated inhibition of T2Rs and allows the activation of the antimicrobial response. (B) Proposed mechanism for T2R and T1R signaling in sinonasal chemosensory cells. T2R signaling is dependent upon $\mathrm{G} \alpha$ gustducin and $\mathrm{IP}_{3} \mathrm{R}$ calcium release channels that likely include the $\mathrm{IP}_{3} \mathrm{R} 3$ isoform. T1R signaling likely uses an alternative $G$ protein that acts through cAMP/PKA and may inhibit IP ${ }_{3}$ R3-mediated calcium signaling. als ( $\sim 0.5 \mathrm{mM} ; \sim 10$-fold less than serum concentration) (46). This low level is nonetheless sufficient to partially inhibit T2R-mediated antimicrobial secretion (Figure 4C). We hypothesized that the T1R2/3-mediated inhibition of T2Rs may exist to modulate the full release of AMPs during times of relative health. However, in the setting of an acute bacterial infection, when bacteria may consume available glucose, causing a sudden decrease in glucose, the T1R2/3 is not activated, removing the inhibition on the T2R response, resulting in an appropriate secretion of AMPs (Figure 8). Alternatively, since the nasal SCCs are diffusely distributed throughout the upper respiratory epithelium (Supplemental Figure 5B), they may control localized bacterial overproliferation by release of AMPs in discrete and localizing regional domains. Thus, the presence of glucose in the ASL acts as a control point in activation of this innate immune defensive response, since complete secretion and depletion of AMPs may leave the epithelium excessively vulnerable during the refractory period (Figure 5C and Figure 6F). That being said, we've demonstrated that priming the system with TLR stimulation upregulates the local production of AMPs, such that SCC T2R stimulation does not completely deplete the defensive reserves, as seen in the absence of TLR priming (Supplemental Figure 17). However, certain chronic conditions, such as diabetes mellitus, have been shown to result in a chronically elevated ASL glucose concentration (46) that may overactivate T1R2/3 and chronically inhibit T2R activation, resulting in reduced AMP secretion. Our data suggest that glucose concentrations of $1.5 \mathrm{mM}$ and above can completely inhibit T2R-mediated AMP secretion, which would likely prevent efficient bacterial killing in an infected airway.

To our knowledge, our data reveal the first demonstrated functional role for T1R sweet receptors in the human airway. While the concentrations of glucose in this study are lower than the concentrations known to stimulate T1R2/3-dependent sweet taste (56) or T1R2/3 activation in heterologous expression systems (57), there is evidence that extraoral sweet receptors can respond to sugar concentrations below taste thresholds in gut endocrine (58) and pancreatic $\beta$ cells (59). Further work must be directed at examining differences in receptor sensitivity and whether there is a role for alternate splicing, posttranslational modifications, and/or accessory subunits.

Based on heterologous expression data, the receptors T2R10, T2R46, and T2R47 are activated by both denatonium and absinthin $(16,60)$. Two other agonists that activate $\beta$-defensin secretion are parthenolide, which activates T2R10 and 46, and amarogentin, which activates T2R46 and 47 (60). A role for T2R47 in this response is in agreement with its localization to solitary nonciliated cells (Figure 2 and Supplemental Figure 7). Future work must be aimed at elucidating the role of these receptors by correlating genetic polymorphisms with the antimicrobial response as well as screening the responses of these receptors to bacterial products. However, we must use caution when comparing the data here with pharmacological profiles obtained during heterologous expression. While heterologous expression generally correlates with taste thresholds, it has been shown that most T2R isoforms form hetero-oligomers with other isoforms (61); oligomerization of sinonasal T2Rs with other T2Rs or other receptors types may change their sensitivities and/or agonist repertoires, as seen with sugar sensitivity differences observed between oral and extraoral $\mathrm{T} 1 \mathrm{R}$ receptors, as described above.

Another important insight provided by our data is that T2Rmediated epithelial AMP secretion is unique to the human upper airway. The T2R-initiated and T1R2/3-regulated calcium signaling 
appears to be similar in human and murine nasal cultures. However, while denatonium-responsive T2R signaling is likely linked to activation of trigeminal neurons in the mouse (11), our human in vitro and ex vivo paradigms suggest that T2R signaling is linked to a more local response that regulates innate immunity. Unfortunately, this also suggests that in vivo studies of this pathway will require the identification and use of a different animal model that better recapitulates human sinonasal physiology, potentially the rabbit (62), pig (63), or sheep (64). For now, differentiated sinonasal epithelial ALI cultures are the only models available to study this response. Our data cannot rule out a similar trigeminally mediated response in humans and mice, suggesting only that the localized immune response via release of AMPs is not present in the mouse. The role of SCC trigeminal activation in humans awaits further investigation.

These data further suggest a potentially important pathophysiological mechanism for increased sinonasal bacterial infection in CRS. If the initial mucosal immune response fails during acute infection, epithelial damage brought about by chronic infection and inflammation may result in increased glucose concentration in nasal ASL, resulting in impairment of the T2R antimicrobial responses and, thus, a positive feedback loop similar to the vicious cycle of infection, inflammation, and tissue damage seen in respiratory diseases, such as cystic fibrosis and chronic bronchitis. These data may also explain why hyperglycemic diabetic patients, who were previously shown to have higher nasal glucose concentrations (46), are more prone to airway infections than nondiabetics (65). If disease progression is accelerated by inhibition of the T2R-mediated antimicrobial secretion, this could lead to identification of novel therapeutic agents. For example, stronger stimulation of the T2R-dependent pathway using bitter agonists and/or inhibition of the T1R2/3 receptor by topical application of compounds like lactisole may restore the ability of sinonasal epithelial cells to mount an appropriate antimicrobial response.

\section{Methods}

Reagents and experimental solutions. Fluo-4, Lucifer yellow biocytin, and the LIVE/DEAD BacLight Bacterial Viability Kit were from Invitrogen. FSL-1 and TLR4 blocking antibody as well as MyD88 and WT littermate mice were gifts from D. LaRosa (University of Pennsylvania). ELISAs for human $\beta$-defensins 1 and 2 (Adipo Biosciences and Peprotech), mouse $\beta$-defensins (MyBioSource), and IL-8 (Pierce Biotechnology) as well as colormetric glucose assay (Cayman Chemical) were all performed according to the manufacturer's instructions. The Luminex 18-Plex Kit was used as previously described (66). Gap 27 was from Tocris Bioscience/R\&D Systems. LPS from $P$. aeruginosa, $18 \alpha-$ GA, and rabbit polyclonal anti-human T1R3 antibody were from Sigma-Aldrich. Polyclonal rabbit antibodies to human $\beta$-defensins 1, 2, and 3 were purchased from Peprotech. Goat polyclonal anti-human T1R2 and T2R47 antibodies, T2R47 blocking peptide, and mouse monoclonal anti- $\beta$-tubulin IV were from Abcam. Mouse monoclonal anti-human $\mathrm{IP}_{3} \mathrm{R} 3$ was purchased from $\mathrm{BD}$ Biosciences. Unless otherwise indicated, other reagents were from Sigma-Aldrich. Experiments were performed with Dulbecco's phosphate-buffered saline (DPBS) on the apical side of cultures and HEPES-buffered Hank's balanced salt solution (10) or Krebs bicarbonate buffer $(30,67)$ on the basolateral side.

Tissue acquisition. Tissue was obtained from patients recruited from the Department of Otorhinolaryngology, Head and Neck Surgery, Division of Rhinology, University of Pennsylvania, and the Philadelphia Veterans Affiairs Medical Center, with informed consent and full approval of both Institutional Review Boards. Patients undergoing sinonasal surgery met the selection criterion for recruitment. Exclusion criteria included a history of systemic diseases (e.g., Wegener's, sarcoid, cystic fibrosis, immunodeficiencies) and use of antibiotics, oral corticosteroids, or antibiologics (e.g., Xolair) within 1 month of surgery. Tissue for establishing ALI cultures or experiments with acutely dissociated cells was obtained from the anterior ethmoid cavity, while tissue for experiments focusing on whole explants was obtained from inferior turbinate. Comparison of mature ALI cultures derived from patients with CRS (with or without nasal polyps) or patients without CRS demonstrated no significant differences in T2R activation or T1R2/3 inhibition of calcium propagation or AMP secretion (data not shown), thus residual clinical material obtained from both patients with and without CRS was used for these studies. Nasal secretions were obtained in the absence of any topical anesthetic or decongestant by placing a preexpanded Pope ear wick (Medtronic ENT) in the nasal cavity under direct visualization between the nasal septum and inferior turbinate bilaterally. Wicks were removed after 5 minutes and placed into an Eppendorf tube. A hole was made in the bottom of the Eppendorf tube with a 20-gauge needle, and the Eppendorf tube was placed inside another Eppendorf tube and centrifuged (1,000 g; 5 minutes); secretions were collected in the outside Eppendorf tube and processed for glucose by colormetric assay. No differences were observed in nasal secretion glucose concentrations between left and right nostrils (data not shown) or between asleep and awake patients (data not shown).

Sinonasal ALI cultures. Sinonasal mucosal specimens were acquired from residual clinical material obtained during sinonasal surgery. ALI cultures were established from enzymatically dissociated HSECs, as previously described $(68,69)$, and grown to confluence with proliferation medium consisting of DMEM/Ham's F-12 and bronchial epithelial basal medium (BEBM; Clonetics) supplemented with $100 \mathrm{U} / \mathrm{ml}$ penicillin and $100 \mu \mathrm{g} / \mathrm{ml}$ streptomycin for 7 days. Cells were then trypsinized and seeded on porous polyester membranes (about $6 \times 10^{4}$ to $7 \times 10^{4}$ cells per membrane) in cell culture inserts (Transwell-clear, $12-\mathrm{mm}$ diameter, $0.4-\mu \mathrm{m}$ pores; Corning) coated with $100 \mu \mathrm{l}$ coating solution (BSA [0.1 mg/ml; Sigma-Aldrich], type I bovine collagen $[30 \mu \mathrm{g} / \mathrm{ml} ; \mathrm{BD}]$, and fibronectin $[10 \mu \mathrm{g} / \mathrm{ml} ; \mathrm{BD}]$ in LHC basal medium [Invitrogen]). Five days later, the culture medium was removed from the upper compartment and the epithelium was allowed to differentiate by using the differentiation medium consisting of 1:1 DMEM (Invitrogen) and BEBM (Clonetics, Cambrex), with the Clonetics complements for hEGF $(0.5 \mathrm{ng} / \mathrm{ml})$, epinephrine $(5 \mu \mathrm{g} / \mathrm{ml})$, BPE $(0.13 \mathrm{mg} / \mathrm{ml})$, hydrocortisone $(0.5 \mu \mathrm{g} / \mathrm{ml})$, insulin $(5 \mu \mathrm{g} / \mathrm{ml})$, triiodothyronine $(6.5 \mu \mathrm{g} / \mathrm{ml})$, and transferrin $(0.5 \mu \mathrm{g} / \mathrm{ml})$, supplemented with $100 \mathrm{UI} / \mathrm{ml}$ penicillin, $100 \mathrm{~g} / \mathrm{ml}$ streptomycin, $0.1 \mathrm{nM}$ retinoic acid (Sigma-Aldrich), and 10\% FBS (Sigma- Aldrich) in the basal compartment. Human bronchial epithelial cells (Lonza) were similarly cultured, as previously described $(70,71)$. Mouse (C57BL) septal ALI cultures were cultured, as previously described (72), from tissue obtained with approval from the University of Pennsylvania and Philadelphia VA Medical Center Institutional Animal Care and Use Committees. Lysates of ALI cultures were made using a lysis buffer containing $150 \mathrm{mM} \mathrm{NaCl}, 1 \mathrm{mM}$ EDTA, $1 \%$ Triton X-100, and $100 \mathrm{mM}$ Tris, pH 7.4, with Roche Complete Protease Inhibitor Cocktail plus PMSF. All lysates were normalized by addition of excess lysis buffer to a protein concentration of $2 \mathrm{mg} / \mathrm{ml}$, measured using a Bio-Rad DC protein assay.

Intracellular calcium and $\mathrm{pH}$ imaging. Fluo-4 loading and imaging were done exactly as previously described (10). Cells were loaded with $10 \mu \mathrm{M}$ Fluo-4-AM (PBS plus 0.1\% pluronic F127; apical side only) for approximately 120 minutes at room temperature, followed by 3 washes with DPBS and 15 to 20 minutes of incubation to allow cells to recover. Images were captured at 3- or 5-second intervals using the $488 \mathrm{nM}$ laser line of an Olympus Fluoview confocal system attached to an Olympus IX81 microscope ( $\times 10,0.3$ NA UPlanFLN objective; Olympus). No gain, offset, or $\gamma$ 
alterations were used. Normalization of Fluo-4 fluorescence changes was made after subtraction of background, approximated for each experiment by measuring unloaded ALIs. Baseline fluorescence $\left(F_{o}\right)$ was determined by averaging the first 10 frames of each experiment. ALIs were loaded with SNARF-5F-AM, as previously described $(30,67)$. Cells were imaged in the absence of $\mathrm{HCO}_{3}^{-}$(20 $\mathrm{mM}$ HEPES basolateral buffering).

Measurement of $C B F$. Cultures were imaged using a $\times 20(0.8$ NA) objective lens on an inverted Leica microscope using a high-speed (100 frames per $\mathrm{s}$ ) Basler $602 \mathrm{f}$ camera and the Sisson-Ammons Video Analysis system (73). All experiments were performed at approximately $28^{\circ} \mathrm{C}$ to $30^{\circ} \mathrm{C}$.

Bacteria culture and bacterial kill assays. P. aeruginosa (strain PAO-1) and K. pneumoniae (strain KP4) were grown in Luria Bertani (LB) medium. MRSA (M2-A9) and S. epidermis (ATCC 12228) were grown in tryptic soy broth. Live-dead staining was performed as previously described (10). Twenty-four hours prior to all assays, sinonasal ALI cultures were washed 3 times with PBS (apical and basolateral side) and then transferred into antibiotic-free, serum-free media (50\% DMEM, 50\% BEBM; containing no antimicrobial agents) on the basolateral side (apical side aspirated dry). Approximately 1 to 2 hours before the assay, basolateral media was again changed, and the apical side was washed 4 times with $300 \mu \mathrm{l}$ PBS. Cultures were then overlaid with $30 \mu \mathrm{l}$ of $50 \%$ PBS with or without various agonists or inhibitors as indicated above. After 30 -minute incubation at $37^{\circ} \mathrm{C}$, the apical solution was carefully removed (to avoid removing any cells), and $20 \mu \mathrm{l} \mathrm{ASL}$ was mixed with $20 \mu \mathrm{l}$ log-phase bacteria (grown overnight in $100 \%$ media, diluted to 0.0001 in $25 \%$ media, and incubated for 1 hour at $37^{\circ} \mathrm{C}$ with shaking) in one well of a 96 -well plate. After shaking on a titer plate shaker $\left(5,000 \mathrm{rpm}\right.$ for 1 minute), the plates were incubated at $37^{\circ} \mathrm{C}$ for 2 hours with shaking every 30 minutes. Three 10 -fold serial dilutions were then made using $25 \%$ bacterial media $\left(10^{0}, 10^{-1}, 10^{-2}, 10^{-3} \mathrm{CFUs}\right)$ and spotted on LB or tryptic soy broth plates. CFUs were counted using the appropriate dilution, in which approximately 10 to 30 colonies could be observed. Often, no colonies were observed in the case of denatonium- or absinthin-treated ASL, which was thus scored as 0 . As there was variability in the number of raw CFUs from experiment to experiment depending on the bacterial culture, results from each experiment were normalized to the percentage CFUs remaining by comparing experimental conditions with the number of CFUs recovered when ASL from unstimulated cultures from the same patients (PBS only) was mixed with bacteria. For determination of salt sensitivity of AMPs, ALIs were stimulated with $10 \mathrm{mM}$ denatonium in $50 \% \mathrm{PBS}(67.5 \mathrm{mM} \mathrm{NaCl})$. ASL was collected and pooled from multiple cultures from each patient and then was aliquoted, with $\mathrm{NaCl}$ added back to 100,135 , or $170 \mathrm{mM}$.

Glucose concentration measurements from sinonasal ALIs. $30 \mu \mathrm{l}$ PBS was placed on the apical side of ALI cultures for 24 hours, assuming that glu- cose concentration would equilibrate during this time and that the concentration would reflect the concentration found in the physiological ASL generated by these cultures. Supporting this, glucose concentrations were also measured at 36 and 48 hours and were not significantly different.

Immunofluorescence microscopy. ALI cultures were fixed for 20 minutes in $4 \%$ paraformaldehyde at $4^{\circ} \mathrm{C}$. The Transwell support was washed 3 times with PBS prior to excision of the membrane containing the cells. The membrane was immersed in Tris-buffered saline with $0.3 \%$ Triton X-100, 5\% normal donkey serum, and $1 \%$ BSA for 60 minutes at room temperature. Primary antibody incubation was performed overnight at $4{ }^{\circ} \mathrm{C}$. Visualization was carried out with Alex Fluor-conjugated donkey anti-mouse IgG (1:500; 75 minutes of incubation at room temperature). Confocal images were acquired with an Olympus Fluoview System (Z-axis step $0.5 \mu \mathrm{m}$; sequential scanning). $\beta$-Defensin staining and T2R47/T1R3/IP 33 costaining were performed with $0.2 \%$ saponin during blocking and antibody incubation steps.

Statistics. Statistical analyses were performed in Excel (Student's $t$ test) and/or GraphPad Prism (Student's $t$ test, ANOVA, and related post-tests). ANOVA was used for comparing multiple data sets. When ANOVA denoted significant differences, post-tests were used to determine individual significance, as indicated in figure legends. Bonferroni post-test was used when preselected pairwise comparisons were performed, Tukey-Kramer post-test was used when all values in the data set were compared, and Dunnett posttest was used when all values were compared with a control value. $P<0.05$ was considered statistically significant.

\section{Acknowledgments}

We thank D.M. Sabatini, G.K. Beauchamp, and D.R. Reed for helpful discussions and suggestions; W. Meyerhof and D.R. Reed for absinthin; P. Jiang for T2R47-transfected HEK293 cells; and D. LaRosa for Myd88 knockout mice. This work was supported by grant 082478 from Flight Attendants Medical Research Institute (to N.A. Cohen), a philanthropic contribution from the RLG Foundation Inc. (to N.A. Cohen), and NIH grant DC03055 (to R.F. Margolskee).

Received for publication July 11, 2013, and accepted in revised form December 11, 2013.

Address correspondence to: Noam Cohen, Perelman School of Medicine at the University of Pennsylvania, Otorhinolaryngology - Head and Neck Surgery, Ravdin Building 5th floor, 3400 Spruce Street, Philadelphia, Pennsylvania 19104, USA. Phone: 215.823.5800, ext.3892; Fax: 215.349.5977; E-mail: cohenn@uphs.upenn.edu.
1. Antunes MB, Gudis DA, Cohen NA. Epithelium, cilia, and mucus: their importance in chronic rhinosinusitis. Immunol Allergy Clin North Am. 2009; 29(4):631-643

2. Tecle T, Tripathi S, Hartshorn KL. Review: Defensins and cathelicidins in lung immunity. Innate Immun. 2010;16(3):151-159.

3. Cherry DK, Woodwell DA. National Ambulatory Medical Care Survey: 2000 summary. Adv Data. 2002;328(328):1-32.

4. Blackwell D, Collins J, Coles R. Summary health statistics for U.S. adults: National Health Interview Survey, 1997. Vital Health Stat. 2002;(205):1-109.

5 . Ray NF, et al. Healthcare expenditures for sinusitis in 1996: contributions of asthma, rhinitis, and other airway disorders. J Allergy Clin Immunol. 1999;103(3 pt 1):408-414.

6. Ly N, McCaig LF. National Hospital Ambulatory Medical Care Survey: 2000 outpatient department summary. Adv Data. 2002;327(327):1-27.
7. Behrens M, Meyerhof W. Bitter taste receptor research comes of age: From characterization to modulation of TAS2Rs. Semin Cell Dev Biol. 2013; 24(3):215-221

8. Lee RJ, Cohen NA. The emerging role of the bitter taste receptor T2R38 in upper respiratory infection and chronic rhinosinusitis. Am J Rhinol Allergy. 2013;27(4):283-286.

9. Shah AS, Ben-Shahar Y, Moninger TO, Kline JN, Welsh MJ. Motile cilia of human airway epithelia are chemosensory. Science. 2009;325(5944):1131-1134.

10. Lee RJ, et al. T2R38 taste receptor polymorphisms underlie susceptibility to upper respiratory infection. J Clin Invest. 2012;122(11):4145-4159.

11. Tizzano M, et al. Nasal chemosensory cells use bitter taste signaling to detect irritants and bacterial signals. Proc Natl Acad Sci U S A. 2010; 107(7):3210-3215.

12. Braun T, Mack B, Kramer MF. Solitary chemosensory cells in the respiratory and vomeronasal epi- thelium of the human nose: a pilot study. Rhinology. 2011;49(5):507-512.

13. Barham HP, et al. Solitary chemosensory cells and bitter taste receptor signaling in human sinonasal mucosa. Int Forum Allergy Rhinol. 2013;3(6):450-457.

14. Tizzano M, Cristofoletti M, Sbarbati A, Finger TE. Expression of taste receptors in solitary chemosensory cells of rodent airways. BMC Pulm Med. 2011;11:3.

15. Behrens M, Meyerhof W. Gustatory and extragustatory functions of mammalian taste receptors. Physiol Behav. 2011;105(1):4-13.

16. Meyerhof W, et al. The molecular receptive ranges of human TAS2R bitter taste receptors. Chem Senses. 2010;35(2):157-170.

17. Gulbransen BD, Clapp TR, Finger TE, Kinnamon SC. Nasal solitary chemoreceptor cell responses to bitter and trigeminal stimulants in vitro. J Neurophysiol. 2008;99(6):2929-2937.

18. Behrens M, Meyerhof W. Mammalian bitter taste perception. Results Probl Cell Differ. 2009;47:203-220. 
19. Palmer RK, et al. Triphenylphosphine oxide is a potent and selective inhibitor of the transient receptor potential melastatin-5 ion channel. Assay Drug Dev Technol. 2010;8(6):703-713.

20. Ohmoto M, Yamaguchi T, Yamashita J, Bachmanov AA, Hirota J, Matsumoto I. Pou2f3/Skn-1a is necessary for the generation or differentiation of solitary chemosensory cells in the anterior nasal cavity. Biosci Biotechnol Biochem. 2013;77(10):2154-2156.

21. Finger TE, Bottger B, Hansen A, Anderson KT, Alimohammadi H, Silver WL. Solitary chemoreceptor cells in the nasal cavity serve as sentinels of respiration. Proc Natl Acad Sci U S A. 2003; 100(15):8981-8986.

22. Ohmoto M, Matsumoto I, Yasuoka A, Yoshihara Y, Abe K. Genetic tracing of the gustatory and trigeminal neural pathways originating from T1R3-expressing taste receptor cells and solitary chemoreceptor cells. Mol Cell Neurosci. 2008;38(4):505-517.

23. Miyoshi MA, Abe K, Emori Y. IP(3) receptor type 3 and PLCbeta2 are co-expressed with taste receptors T1R and T2R in rat taste bud cells. Chem Senses. 2001;26(3):259-265.

24. Hisatsune C, et al. Abnormal taste perception in mice lacking the type 3 inositol 1,4,5-trisphosphate receptor. J Biol Chem. 2007;282(51):37225-37231.

25. Jiang $P$, et al. Lactisole interacts with the transmembrane domains of human T1R3 to inhibit sweet taste. J Biol Chem. 2005;280(15):15238-15246.

26. Cui M, Jiang P, Maillet E, Max M, Margolskee RF, Osman R. The heterodimeric sweet taste receptor has multiple potential ligand binding sites. Curr Pharm Des. 2006;12(35):4591-4600.

27. Imada T, Misaka T, Fujiwara S, Okada S, Fukuda $\mathrm{Y}$, Abe K. Amiloride reduces the sweet taste intensity by inhibiting the human sweet taste receptor. Biochem Biophys Res Commun. 2010;397(2):220-225.

28. Behrens M, et al. Immunohistochemical detection of TAS2R38 protein in human taste cells. PLoS One. 2012;7(7):e40304.

29. Antunes MB, et al. Murine nasal septa for respiratory epithelial air-liquid interface cultures. Biotechniques. 2007;43(2):195-204.

30. Lee RJ, Harlow JM, Limberis MP, Wilson JM, Foskett JK. HCO3(-) secretion by murine nasal submucosal gland serous acinar cells during Ca2+-stimulated fluid secretion. J Gen Physiol. 2008;132(1):161-183.

31. Lee RJ, et al. Vasoactive intestinal peptide regulates sinonasal mucociliary clearance and synergizes with histamine in stimulating sinonasal fluid secretion. FASEB J. 2013;27(12):5094-5103.

32. Margolskee RF. Molecular mechanisms of bitter and sweet taste transduction. J Biol Chem. 2002;277(1):1-4.

33. Sbarbati A, et al. Identification and characterization of a specific sensory epithelium in the rat larynx. J Comp Neurol. 2004;475(2):188-201.

34. Giovannucci DR, Groblewski GE, Sneyd J, Yule DI. Targeted phosphorylation of inositol 1,4,5-trisphosphate receptors selectively inhibits localized $\mathrm{Ca} 2+$ release and shapes oscillatory $\mathrm{Ca} 2+$ signals. J Biol Chem. 2000;275(43):33704-33711.

35. Yule DI, Straub SV, Bruce JI. Modulation of Ca2+ oscillations by phosphorylation of $\operatorname{Ins}(1,4,5) \mathrm{P} 3$ receptors. Biochem Soc Trans. 2003;31(pt 5):954-957.

36. Prince A. The bitter taste of infection. J Clin Invest. 2012;122(11):3847-3849.

37. Carothers DG, et al. Production of beta-defensin antimicrobial peptides by maxillary sinus mucosa. Am J Rhinol. 2001;15(3):175-179.
38. Singh PK, Tack BF, McCray PB, McCray PB Jr, Welsh MJ. Synergistic and additive killing by antimicrobial factors found in human airway surface liquid. Am J Physiol Lung Cell Mol Physiol. 2000;279(5):L799-L805.

39. Bals R, et al. Human beta-defensin 2 is a salt-sensitive peptide antibiotic expressed in human lung. J Clin Invest. 1998;102(5):874-880.

40. Guani-Guerra E, Negrete-Garcia MC, Montes-Vizuet R, Asbun-Bojalil J, Teran LM. Human beta-defensin- 2 induction in nasal mucosa after administration of bacterial lysates. Arch Med Res. 2011;42(3):189-194.

41. Thienhaus ML, Wohlers J, Podschun R, Hedderich J, Ambrosch P, Laudien M. Antimicrobial peptides in nasal secretion and mucosa with respect to Staphylococcus aureus colonization in chronic rhinosinusitis with nasal polyps. Rhinology. 2011;49(5):554-561.

42. Parker D, Prince A. Innate immunity in the respiratory epithelium. Am J Respir Cell Mol Biol. 2011;45(2):189-201.

43. Ooi EH, Wormald PJ, Carney AS, James CL, Tan LW. Fungal allergens induce cathelicidin LL-37 expression in chronic rhinosinusitis patients in a nasal explant model. Am J Rhinol. 2007;21(3):367-372.

44. Ooi EH, Wormald PJ, Tan LW. Innate immunity in the paranasal sinuses: a review of nasal host defenses. Am J Rhinol. 2008;22(1):13-19.

45. Woods CM, et al. Lysozyme expression is increased in the sinus mucosa of patients with chronic rhinosinusitis. Rhinology. 2012;50(2):147-156.

46. Garnett JP, Baker EH, Baines DL. Sweet talk: insights into the nature and importance of glucose transport in lung epithelium. Eur Respir J. 2012;40(5):1269-1276

47. Kalsi KK, et al. Glucose homeostasis across human airway epithelial cell monolayers: role of diffusion, transport and metabolism. Pflugers Arch. 2009;457(5):1061-1070

48. Kalsi KK, et al. Apical and basolateral localisation of GLUT2 transporters in human lung epithelial cells. Pflugers Arch. 2008;456(5):991-1003.

49. Pezzulo AA, et al. Glucose depletion in the airway surface liquid is essential for sterility of the airways. PLoS One. 2011;6(1):e16166.

50. Philips BJ, et al. Glucose in bronchial aspirates increases the risk of respiratory MRSA in intubated patients. Thorax. 2005;60(9):761-764.

51. Baker EH, et al. Hyperglycemia and cystic fibrosis alter respiratory fluid glucose concentrations estimated by breath condensate analysis. J Appl Physiol. 2007;102(5):1969-1975.

52. Garnett JP, et al. Proinflammatory mediators disrupt glucose homeostasis in airway surface liquid. J Immunol. 2012;189(1):373-380.

53. Soyka MB, et al. Defective epithelial barrier in chronic rhinosinusitis: the regulation of tight junctions by IFN- $\gamma$ and IL-4.J Allergy Clin Immunol. 2012;130(5):1087-1096

54. Rogers GA, Den Beste K, Parkos CA, Nusrat A, Delgaudio JM, Wise SK. Epithelial tight junction alterations in nasal polyposis. Int Forum Allergy Rhinol. 2011;1(1):50-54.

55. Masubuchi Y, et al. A novel regulatory function of sweet taste-sensing receptor in adipogenic differentiation of 3T3-L1 cells. PLoS One. 2013;8(1):e54500.

56. Schiffman SS, Booth BJ, Sattely-Miller EA, Graham BG, Gibes KM. Selective inhibition of sweetness by the sodium salt of +/-2-(4-methoxyphenoxy)propanoic acid. Chem Senses. 1999;24(4):439-447.

57. Li X, Staszewski L, Xu H, Durick K, Zoller M, Adler
E. Human receptors for sweet and umami taste. Proc Natl Acad Sci US A. 2002;99(7):4692-4696.

58. Jang HJ, et al. Gut-expressed gustducin and taste receptors regulate secretion of glucagonlike peptide-1. Proc Natl Acad Sci U S A. 2007; 104(38):15069-15074.

59. Kyriazis GA, Soundarapandian MM, Tyrberg B. Sweet taste receptor signaling in beta cells mediates fructose-induced potentiation of glucose-stimulated insulin secretion. Proc Natl Acad Sci US A. 2012; 109(8):E524-E532.

60. Wiener A, Shudler M, Levit A, Niv MY. BitterDB: a database of bitter compounds. Nucleic Acids Res. 2012;40(Database issue):D413-D419.

61. Kuhn C, Bufe B, Batram C, Meyerhof W. Oligomerization of TAS2R bitter taste receptors. Chem Senses. 2010;35(5):395-406.

62. Chiu AG, Antunes MB, Feldman M, Cohen NA. An animal model for the study of topical medications in sinusitis. Am J Rhinol. 2007;21(1):5-9.

63. Wang JC, Hathorn I, Habib AR, Chang E, Javer AR. Evaluation of domestic and Yucatan swine nasal sinus anatomy as models for future sinonasal research of medications delivered by standard instruments used in functional endoscopic sinus surgery. Int Forum Allergy Rhinol. 2013;3(2):150-156.

64. Ha KR, Psaltis AJ, Tan L, Wormald PJ. A sheep model for the study of biofilms in rhinosinusitis. Am J Rhinol. 2007;21(3):339-345.

65. Koziel H, Koziel MJ. Pulmonary complications of diabetes mellitus. Pneumonia. Infect Dis Clin North Am. 1995;9(1):65-96.

66. Hekiert AM, et al. Biofilms correlate with TH1 inflammation in the sinonasal tissue of patients with chronic rhinosinusitis. Otolaryngol Head Neck Surg. 2009;141(4):448-453.

67. Kreindler JL, et al. Interleukin-17A induces bicarbonate secretion in normal human bronchial epithelial cells. Am J Physiol Lung Cell Mol Physiol. 2009;296(2):L257-L266.

68. Lai Y, Chen B, Shi J, Palmer JN, Kennedy DW, Cohen NA. Inflammation-mediated upregulation of centrosomal protein 110, a negative modulator of ciliogenesis, in patients with chronic rhinosinusitis. J Allergy Clin Immunol. 2011;128(6):1207-1215.

69. Ramanathan M, Ramanathan M Jr, Lane AP. A comparison of experimental methods in molecular chronic rhinosinusitis research. Am J Rhinol. 2007; 21(3):373-377.

70. Savitski AN, Mesaros C, Blair IA, Cohen NA, Kreindler JL. Secondhand smoke inhibits both $\mathrm{Cl}-$ and $\mathrm{K}+$ conductances in normal human bronchial epithelial cells. Respir Res. 2009;10:120.

71. Zhao KQ, Xiong G, Wilber M, Cohen NA, Kreindler JL. A role for two-pore $\mathrm{K}+$ channels in modulating $\mathrm{Na}+$ absorption and $\mathrm{Cl}$ - secretion in normal human bronchial epithelial cells. Am J Physiol Lung Cell Mol Physiol. 2012;302(1):L4-L12.

72. Antunes MB, et al. Murine nasal septa for respiratory epithelial air-liquid interface cultures. Biotechniques. 2007;43(2):195-196.

73. Sisson JH, Stoner JA, Ammons BA, Wyatt TA. All-digital image capture and whole-field analysis of ciliary beat frequency. J Microsc. 2003;211(pt 2):103-111.

74. Wood DM, Brennan AL, Philips BJ, Baker EH. Effect of hyperglycaemia on glucose concentration of human nasal secretions. Clin Sci (Lond). 2004; 106(5):527-533

75. Philips BJ, Meguer JX, Redman J, Baker EH. Factors determining the appearance of glucose in upper and lower respiratory tract secretions. Intensive Care Med. 2003;29(12):2204-2210. 